\title{
Extending the verifiability approach framework: \\ The effect of initial questioning
}

Adam Charles Harvey, Aldert Vrij, George Sarikas, Sharon Leal, Louise Jupe and Galit Nahari

\begin{abstract}
The Verifiability Approach (VA) is a lie detection tool that examines reported checkable details. Across two studies, we attempt to exploit liar's preferred strategy of repeating information by examining the effect of questioning adult interviewees before the VA. In Study 1, truth tellers $(n=34)$ and liars $(n=33)$ were randomly assigned to either an initial open or closed questioning condition. After initial questioning, participants were interviewed using the VA. In Study 2, truth tellers $(n=48)$ and liars $(n=48)$ were interviewed twice, with half of each veracity group randomly assigned to either the Information Protocol (an instruction describing the importance of reporting verifiable details) or control condition. Only truth tellers revised their initial statement and to include verifiable detail. This pattern was most pronounced when initial questioning was open (Study 1) and when the information protocol was used between recall attempts (Study 2). Thus, liar's preferred strategy of maintaining consistency between statements appears exploitable using the VA.
\end{abstract}




\section{General Audience Summary:}

Across two studies we explored if the Verifiability Approach - a technique for distinguishing liars from truth tellers using the quantity of verifiable detail reported in interviewees' statements - can be adapted to elicit consistency-based cues to deception by asking questions before administering the full VA procedure..Truth tellers appeared willing to revise their initial statement and, when prompted, reported previously undisclosed verifiable detail. Conversely, liars appeared unwilling to revise their initial statement to include new verifiable detail.

Key Words: Verifiability Approach; lie detection; between-statement consistency; eliciting cues; verbal cues 
Extending the Verifiability Approach framework:

The effect of initial questioning

Individuals often lie to avoid punishments for their actions (Vrij, 2008). Hence, detecting deception is often central to police investigations (Vrij, Mann, Kristen, \& Fisher, 2007) and court proceedings (Leach et al., 2016). Unfortunately, accurate lie detection is difficult (Bond \& DePaulo, 2006; Vrij \& Granhag, 2012) because there are insufficient reliable veracity cues available to credibility assessors (DePaulo et al., 2003; Hartwig \& Bond, 2011). Hence, the development of interviewing techniques that actively elicit cues to deception is required (Vrij \& Granhag 2014, Vrij, Fisher \& Blank, 2017).

One such technique - the Verifiability Approach (VA; Nahari, Vrij \& Fisher 2014a, $2014 b$ ) - is built upon the assumption that liars are strategically motivated to avoid reporting details that can be checked by investigators. Interviewing techniques that further discourage liars (but not truth tellers) from reporting verifiable information should improve the VA's ability to distinguish liars and truth tellers. The current studies investigate if liar's welldocumented motivation to report consistent information between statements (e.g. Granhag \& Strömwall, 1999; 2001; Granhag, Strömwall, \& Jonsson, 2003) can be exploited by eliciting a preliminary statement from suspects before the VA. Specifically, we examine if liar's attempts to maintain consistency with the information they report before the VA reduces their ability to report checkable information during the VA, compared to truth tellers. Thus, we test the hypothesis that tactically obtaining statements before the VA can improve the ability of the VA to subsequently distinguish liars and truth tellers.

\section{The Verifiability Approach}

The Verifiability Approach (VA) is based on the assumption that during interviewing, liars have two antagonistic motivations. First, liars want to provide detailed statements in 
order to sound credible (Hartwig et al., 2007; Strömwall et al., 2006). This strategy makes sense as more detailed statements are more likely to be perceived as credible (Bell \& Loftus, 1989; Johnson, 2006). Second, liars want to minimise the investigator's opportunities to potentially falsify their statement (Masip \& Herrero, 2013; Nahari et al., 2014a). This strategy also makes sense as investigators compare reported information to evidence (e.g., Hartwig, Granhag, Strömwall \& Vrij, 2005).

According to the VA, a potential solution to this dilemma is for liars to withhold providing details an investigator can verify. Verifiable information is detail that can be checked by the investigator and its veracity determined, such as phone call records. Information that cannot be checked to determine its veracity is unverifiable, such as being at home alone (Nahari et al., 2014a). Truth tellers are more forthcoming than liars (Hartwig, Granhag, \& Strömwall, 2007; Hartwig, Granhag, Strömwall \& Doering, 2010), especially with verifiable details (Nahari et al., 2014a).

The 'Information Protocol' (IP; Nahari et al., 2014b) can amplify these differences further and is an integrated component of the full VA (Nahari \& Vrij, 2014). The IP informs suspects of the importance of including verifiable information in their statements (for a full description, see Harvey, Vrij, Leal, Laffery \& Nahari, 2017). Administering the IP (versus withholding the IP) elicits more verifiable details from truth tellers, but not from liars. Hence, verifiable details have emerged as a promising verbal cue to deception (Nahari et al., 2014b).

\section{Between-statement Consistency}

Between-statement consistency refers to the degree of similarity between separate accounts from a single person (Fisher, Vrij \& Leins, 2013; Vredeveldt, van Koppen \& Granhag, 2014). The belief that between-statement consistency (and inconsistency) indicates honesty (and dishonesty) respectively is referred to as the 'consistency heuristic' (Granhag \& Stromwall, 1999; Stromwall \& Granhag, 2003). Research has shown between-statement 
consistency to be an unreliable cue to deception (Fisher et al., 2013; Vredeveldt et al., 2014), with truth tellers and liars often displaying similar levels of consistency. This similarity, according to the 'Repeat versus Reconstruct Hypothesis', is theoretically due to i) deliberate attempts by liars to repeat previously disclosed information in follow-up statements (increasing their between-statement consistency) and ii) truth tellers' tendency to reconstruct events from memory in follow-up statements (decreasing their between-statement consistency) (Granhag et al., 1999).

Proactive interviewing may exploit these differences in strategy and magnify the difference between liars and truth tellers in terms of between-statement consistency (e.g. Deeb et al., 2017; Leins et al., 2011, 2012). The VA framework may be well-suited for eliciting between-statement consistency as a reliable cue to deception, especially with respect to reported verifiable detail. The established VA protocol employs an IP instruction followed by a free-recall (e.g. Nahari et al., 2014b). Theoretically, when interviewed once before, and once during the VA, truth tellers can respond to the VA's IP instruction by reporting additional verifiable details (i.e. previously unreported information), i.e. in a manner consistent with predictions based upon the 'Repeat versus Reconstruct Hypothesis'. In contrast, liars are likely to be influenced by their motivation to maintain between-statement consistency (Granhag et al., 1999, 2002, 2003) and may thus be less wiling to provide additional verifiable details after an IP instruction.

\section{Questioning styles}

Real-world investigators may decide to commence preliminary questioning before the VA protocol as a tactic to limit the opportunities for liars to use counter-measures (i.e. techniques employed by interviewees to defeat the lie detection tool; Ganis, Rosenfeld, Meixner, Kievit \& Schendan, 2011) such as reporting false verifiable details (Nahari \& Vrij, 2015) in response to the VA's IP instruction. A common distinction made in questioning 
styles is between open and closed questions (Oxburgh, Myklebust \& Grant, 2010; Hartwig, Granhag, Stromwall, Wolf, Vrij, \& Hjelmsäter, 2011). Closed questions typically demand only binary yes-no responses from interviewees ('Did you see anyone?'), and only obtain limited information (Oxburgh et al., 2010). However, open questions allow for unrestricted responses (Home Office, 2007) and are most likely to obtain detailed statements (Myklebust \& Bjorklund, 2006; Lamb et al., 1996). For liars, even detailed statements are unlikely to contain extensive verifiable information (Harvey et al., 2017b).

Revising an initial statement to provide additional verifiable detail is directly antagonistic with the liar's motivations to i) avoid disclosing checkable information (Nahari et al., 2014a) and ii) maintain between-statement consistency (Granhag et al., 1999, 2001, 2003). Thus, eliciting detailed statements via free-recall questioning makes it difficult for liars to revise their initial statement without becoming inconsistent. As such, liars may ignore the VA's IP instruction. In contrast, closed questions typically specifically target information of interest to the investigators (such as the existence of evidence), therefore forcing liars to address critical topics. However, responses to closed questions are typically short, which gives liars opportunities to embellish, revise and explain away their original answer in a subsequent statement without contradiction. For example, a liar may admit to a potentially traceable witness (by responding 'yes' to a closed question), but then uses embedding strategies to revise the subsequent statement and include multiple witnesses. This would allow the liar to provide additional verifiable detail and to maintain between-statement consistency.

We thus predict that liars will report more verifiable detail, and a greater proportion of verifiable detail, when questioned via the VA in the preliminary closed (compared to the preliminary open) condition (Hypothesis 1). In contrast, no difference is expected between the number (or proportion) of verifiable details reported between open or closed preliminary 
questioning conditions for truth tellers (Hypothesis 2). We also predict liars to display greater consistency - reporting fewer Verifiable Source commissions - in the preliminary open (versus closed) questioning condition, whereas no difference in consistency is predicted for truth tellers (Hypothesis 3). Finally, we predict that higher classification rates in discriminating between truth tellers and liars in their final statements (using both the number and proportion of verifiable details [number of verifiable details/ total number of details reported]) will emerge in the preliminary open than in the preliminary closed questioning condition (Hypothesis 4).

\section{Study 1}

\section{Method}

\section{Design}

The study used a 2 (Veracity: lie vs. truth) x 2 (Preliminary Questioning Style) between-subjects design. The dependent measures were: i) consistency of verifiable sources (to assess between-statement consistency of verifiable information); ii) the number of reported verifiable details in the final statement; and iii) the proportion of verifiable details reported in the final statement. All interviewees were interviewed twice: once before the VA and once during the VA.

\section{Participants}

A power analysis using G*Power (Faul, Erdfelder, Lang \& Buchner, 2007), assuming a large effect size of $f=0.4$ (derived from Nahari et al., 2014b; Harvey et al. 2017a) $(\alpha=0.05)$ for four groups, indicated a sample size of 54 (approx. 14 per cell) would be sufficient for an acceptable power of 0.80 (Cohen 1992). Four participants were excluded for not following experimental instructions, leaving a total of 67 volunteers, consisting of 42 females and 25 males, aged between 18 and 29 years $(M=19.52$ years, $S D=1.67,95 \%$ CI $[19.16,19.97]$, range 18-29), from the University's undergraduate and postgraduate communities 
participated in the study in exchange for course credit.

\section{Ethics}

A favorable ethical review decision was given, prior to the research, by the University Science Faculty Ethics Committee (SFEC). Our Institution's SFEC conforms to the British equivalent of APA ethical standards (see British Psychological Society, 2009; 2014). All participants' rights were protected during the study.

\section{Procedure}

Participants were recruited via adverts on the University's online participant pool. We solicited individuals for a study on deception in a police suspect setting. Individuals arrived at the laboratory at pre-arranged times. Each participant was given an information sheet about the study and was asked to sign an informed consent form.

Participants were randomly allocated to either the truthful or deceptive condition. Truth tellers $(n=33)$ were provided the following instructions (replicating those in the previous VA literature, see Nahari et al., 2012; 2014a; 2014b); 'You are requested to do your normal business for 30 minutes. Make sure that you carry out more than one activity and do not stay at one place for more than 10 minutes. Please carry out as many activities as you can, such as buying coffee, having a conversation with a friend, visiting the book shop, etc. Please do not do anything that you may later be unable or uncomfortable to talk about. Please return in exactly 30 minutes (the experimenter indicated the exact time) to the lab for the second stage of the study.' (Note that none of the suggested activities automatically lead to reporting verifiable detail, these instructions are therefore not leading.)

After the participant returned to the laboratory, the experimenter said: 'In the last 30 minutes, a USB device containing sensitive material (forthcoming exam questions) was stolen from one of the offices in the Psychology Department. As you were not with myself or a colleague during this time, you are one of the suspects in this theft. You are going to be 
required to write a statement about your activities during the 30 -minute period. Your task is to be totally honest and provide as much information as you can remember. Your typed statement will be passed to the investigating officer who is responsible for investigating the theft.'

Liars $(n=34)$ were provided the following information: 'Your mission is to commit a 'theft' of the USB device containing the question topics scheduled to be used in an upcoming psychology exam. Because of the sensitive nature of the materials, you should try to accomplish your mission as inconspicuously as possible.' Participants where then provided detailed instructions on how to navigate to a specific room within the psychology department and a description of the device they were to retrieve. These activities took approximately 30 minutes to complete and, once completed, the participant was instructed to return to the experimenter. Upon the participant returned to the laboratory, the experimenter said: 'In the last 30 minutes, a USB device containing sensitive material (forthcoming exam questions) was stolen from one of the offices in the Psychology Department. As you were not with myself or a colleague during this time, you are one of the suspects in this theft. You are going to be asked to write a statement describing what you were doing during the last 30 minutes. Your task is to lie about your activities so that anyone reading your statement will believe you had nothing to do with the theft. Specifically, it is important you deny taking the USB device and instead pretend you've been going about your normal business inside, and/or in the area surrounding, the department for the 30 minutes. Your typed statement will be passed to the investigating officer who is responsible for investigating the theft.'

All participants were allowed as much time as they required to prepare themselves for the interview. Upon indicating they were ready to be interviewed, all participants (truthtellers and liars) were informed that the interview itself would be carried out by an automated computer program and that they should follow the on-screen instructions. The automated 
interview was originally developed as part of efforts to automate the entire Verifiability Approach procedure for use in high-traffic, resource-constrained settings [see Kleinberg, Mozes, Arntz and Verschuere (2017) for research into automating verifiability coding]. We constructed the interview using 'Qualtrics' (www.qualtrics.com), an online survey authorship and software provider. The experimenter informed both truth tellers and liars that their objective was to convince the human investigator (who would read their statement) that they were not responsible for the theft.

Half of the truth tellers and liars were randomly allocated to each of the preliminary probe or free-recall conditions. All suspects were told to follow the on-screen instructions that began by asking interviewees to read all the instructions carefully because, once they progressed, they would be unable to return to a previous page.

Suspects in the preliminary probe questioning condition ( $n=17$ liars, $n=16$ truth tellers) were first asked a series of six closed questions (see Appendix 1). These yes/no forced choice questions assessed systematically if any of the suspects activities for the previous 30 minutes had been, in fact, verifiable. Suspects selected their response from the two options before the next question was presented. Once all six preliminary probe questions had been answered, the program stated the interview would begin.

Suspects in the preliminary open questioning condition ( $n=17$ liars, $n=17$ truth tellers) were first asked to provide, in as much detail as possible, a statement regarding their activities during the previous 30 minutes (i.e. from the time they left the laboratory to the time they returned). Suspects typed their response and once they indicated they were finished, the program stated the interview would begin.

After the preliminary questioning (closed or open), all interviews were identical: interviewees were provided the IP instruction (see Nahari et al., 2014b) that was provided on screen. Participants were told to read carefully the IP instruction (Appendix 2) and then once 
completed, they would be asked one final question.

After the IP instruction, the program requested suspects to type, in as much detail as possible, their statement. Once suspects indicated they were finished writing their statement, they were instructed to inform to the experimenter they had completed the interview.

After the interview, the participants completed a post-interview questionnaire. The questions included the following manipulation check: 'To what extent is your account based on a truthful experience?' Participants were asked to respond on an 11-point Likert scale, ranging from $0 \%$ to $100 \%$ of their statement being based upon a truthful experience. Participants were also asked 'To what extent were you motivated to give an honest impression and appear convincing during your interviews' and to rate their motivation on a 5point Likert scale ranging from 1 (not at all motivated) to 5 (extremely motivated). Participants were also asked to rate, on 5-point Likert scales ranging from 1 (not at all motivated) to 5 (extremely motivated), to what extent they were motivated to provide a detailed statement in the interview. Once completed, participants were debriefed, awarded course credit and thanked for their time.

\section{Coding}

We coded statements for: i) consistency of verifiable sources (to assess betweenstatement consistency of verifiable information), and ii) the number of reported verifiable and unverifiable details in the final statement. From the number of reported verifiable and unverifiable details in the final statement, we also calculated the proportion of verifiable details reported in the final statement (proportion of verifiable detail $=$ number of verifiable details/ [number of verifiable details + number of unverifiable details]).

\section{Coding the verifiability source consistency.}

We distinguished between six sources that contribute to the potential verifiability of a suspect's statement (see Table 1). Each verifiable source corresponded to a preliminary 
closed question so a 'yes' response was coded as 'source present' whereas a 'no' was coded as 'source absent'. For the open questions we coded a verifiable source present if any of the verifiable information in the statement corresponded to it. For example, the statement '...$I$ met with my friend Ludwig and then bought lunch using my debit card...' corresponds to two verifiable sources: 'met with a traceable other' and 'electronic evidence'. Similarly, the statement ' ...I bought a bus ticket and phoned my house mate to say I was coming back. On the bus, I sat next to and chatted with my lecturer Professor Wittgenstein and watched myself on the buses CCTV screen...' corresponds to four verifiable sources: 'physical evidence', 'phone evidence', 'witnessed by a traceable other' and 'CCTV evidence'.

Thus, a participant's verifiable source score could range from 0 (no verifiable sources) to 6 (six verifiable sources) reported per statement. A second coder was randomly allocated the statements given by a sample of $17(25.4 \%)$ participants and scored them for the occurrence of verifiable sources. Inter-rater reliabilities between the two coders for the verifiable factor score were measured via intra-class correlation coefficients (ICC). The ICC was good and therefore satisfactory for verifiable source score $[\mathrm{ICC}]=.76,95 \% \mathrm{CI}[.46, .91]$ $(p<0.001)$

\section{[Insert Table 1 about here]}

To calculate a sensitive measure of consistency (see Granhag, Stromwall, Willen \& Hartwig, 2012), each verifiable source was classified as either omitted (present in statement 1, absent in statement two); committed (present in statement 2, absent in statement 1); repeated (present in both statements and corresponding) or contradicted (present in both statements and conflicting) (see Fisher et al., 2013; Vredeveldt et al., 2014). Contradictions did not occur in our data, so only analysis of omissions, commissions and repetitions are presented. A second coder was randomly allocated the statements given by a sample of 17 (25.4\%) participants and scored them for the occurrence of verifiable sources. Inter-rater 
reliabilities between the two coders for the verifiable factor score were measured via intraclass correlation coefficients (ICC). The ICC was good and therefore satisfactory for verifiable source omissions $[\mathrm{ICC}]=.82,95 \% \mathrm{CI}[.50, .93](p<0.001)$, verifiable source commissions $[\mathrm{ICC}]=.94,95 \% \mathrm{CI}[.73, .96](p<0.001)$, and verifiable source repetitions $[\mathrm{ICC}]=.89,95 \% \mathrm{CI}[.69, .96](p<0.001)$.

\section{Coding the verifiability of statements and inter-rater reliability.}

Both the main and second (inter-rater) coder have coded extensively within the department previously. The coding protocol adopted was identical to Harvey et al. (2017a; 2017b) and follows the established VA procedure in which reported information is coded for detail and classified as either in principle verifiable or in principle unverifiable.

All statements were first rated by one coder (blind to the experimental conditions) who scored the frequency occurrence of overall detail using interpersonal Reality Monitoring (RM) criteria. RM is frequently used in the deception literature due to its clearly defined criteria, reliability and ability to capture the detail contained with a statement (Vrij, 2008). For clarity, we provide examples of each detail type below, with each unit of analysis underlined. We distinguished between the following criteria (e.g. Nahari et al., 2014a; 2014b; Vrij, 2015): Perceptual detail (information about what was seen, heard, felt and smelt during the described activities; e.g. 'The cleaner waved and said hello), spatial detail (information about locations or the arrangement of persons and/or objects; e.g. 'I walked up the stairs'; 'I sat to the left of Zvi') and temporal details (information about when the event happened and explicit descriptions of the sequence of various events; e.g. 'I then called my girlfriend and after that my house mate'). We did not formulate hypotheses about these different categories of detail, so they were merged into one 'total detail' category. (Coding in different categories was carried out to increase the reliability of the coding.) We then computed total detail criteria (summation of perceptual, spatial and temporal details) that potentially could or could 
not be verified. This resulted in 'information units' that could be a single word or combinations of words, depending upon the meaning (for a discussion of the VA coding protocol, see Nahari 2017). Verifiable details were activities the interviewee said were (i) carried out with (an)other identifiable person(s) who can be traced (e.g. staff at a named establishment: 'The Manager at the Library was informed' or a named person: 'Oيscar Keats was told'), (ii) witnessed by at least one other identifiable and traceable person (e.g. 'the

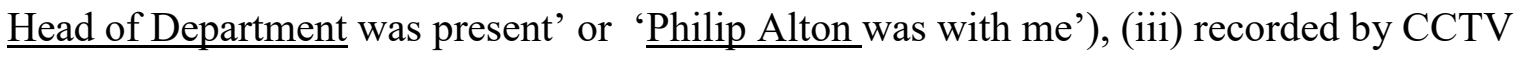
cameras, or (iv) documented and therefore checkable (e.g., phone calls, cash withdrawal from ATM machines; 'the police were phoned'). Details that do not meet at least one of these criteria were classified as unverifiable (Nahari et al., 2014a, 2014b). Importantly, the definitions for verifiable and unverifiable details that were used for coding were identical to those given to the participants in the IP instructions.

A second coder (also blind to the veracity of the statements) coded a random selection of 17 statements ( $\operatorname{circa} 25 \%$ ). Inter-rater reliabilities between the two coders for the occurrence frequency of total detail and for detail that could or could not be verified, were measured via intra-class correlation coefficients (ICC). The ICC was good and therefore satisfactory for total detail $[\mathrm{ICC}]=.90,95 \% \mathrm{CI}[.75, .96](p<0.001)$, verifiable detail [ICC] $=$ $.91,95 \%$ CI $[.77, .97](p<0.001)$, and unverifiable detail [ICC $]=.84,95 \%$ CI $[.61, .94](p<$ $0.001)$.

\section{Supplementary analysis section}

Below are presented the results for the number (and proportion) of verifiable details reported in the final statement, and for the consistency (number of commissions, omissions and repetitions) of verifiable sources. For additional analysis of the number of unverifiable detail reported in the final statement, see the supplementary analysis section.

\section{Results}




\section{Manipulation Checks}

\section{Veracity manipulation check.}

Truthful mock-suspects reported their overall truthfulness rating as high $(M=97.27 \%$, $S D=5.17 \%, 95 \% \mathrm{CI}[95.52 \%, 98.92 \%])$ and deceptive mock-suspects reported a significantly lower truthfulness rating $(M=37.94 \%, S D=34.97 \%, 95 \%$ CI $[26.97 \%$, $49.12 \%]), t(34.484)=9.784, p<0.001, d=2.37,95 \%$ CI $[1.71,2.95]$. This supports the validity of the veracity manipulation.

\section{Motivation.}

The reported motivation of the mock-suspects (henceforth suspects) to perform well was relatively high $(M=3.97, S D=.85,95 \% \mathrm{CI}[3.76,4.12])$ on a 5-point Likert scale. A 2 (Veracity) X 2 (Preliminary Questioning Style) analysis of variance (ANOVA) showed no main effect for Veracity or Preliminary Questioning Style, and no Veracity X Preliminary Questioning Style interaction, all F's $<1$, all $p$ 's $>.680$.

\section{Hypothesis Testing}

To examine how strongly our data supported the hypothesis, we - in addition to reporting NHST - also calculated Bayes Factor (BF) scores (e.g. Wagenmakers et al., 2016). Note that the default Bayesian $t$ test used here is based on general considerations that represent a lack of knowledge about the effect size under study (e.g. Wagenmakers et al., 2011). Table 2 shows the interpretation of Bayes Factor derived from Jeffreys (1961) and adapted by Wagenmakers et al. (2011).

\section{[Table 2 about here]}

\section{Number of verifiable detail in the final statement.}

A 2 (Veracity) x 2 (Preliminary Questioning Style) ANOVA with the number of verifiable details in the final statement as the dependent variable revealed a significant main effect for Veracity, $F(1,63)=21.04, \mathrm{MSE}=1615.29, p<0.001, d=1.11,95 \%$ CI $[.58$, 
1.61]. However, the main effect for Preliminary Questioning Style was not significant, $F(1$, $63)=.82, \mathrm{MSE}=63.07, p=0.368, d=.18,95 \%$ CI $[-.30, .66]$. Furthermore, the Veracity $\mathrm{x}$ Preliminary Questioning Style interaction effect was not significant, $F(1,63)=3.00$, MSE $=$ $229.88, p=0.088, \eta_{p^{2}}=.05$

The interaction statistics $(p=.088)$ refers to any type of interaction. However, in our Hypothesis 1 and 2 we predicted a specific type of interaction: liars will report more verifiable detail in their final statement in the preliminary closed than in the preliminary open questioning condition, whereas verifiable detail will not vary as a function of preliminary question style for truth tellers. Therefore, a more informative test of Hypothesis 1 and 2 is to statistically test for significant differences between the two Preliminary Questioning Style conditions for truth-tellers and liars separately. This approach, introduced by Nahari and Ben-Shakhar (2011), has now been adapted by others (Deeb, Vrij, Hope, Mann, Granhag \& Lancaster, 2016; Nahari, 2017; Shaw, Vrij, Leal, Mann, Hillman, Granhag \& Fisher, 2015). Thus, we examined the difference in the number of verifiable detail reported by truth tellers and liars, for the two preliminary interview style conditions separately, as this addresses Hypothesis 1 and 2 directly.

[Table 3 about here]

As Table 3 shows, liars in the Preliminary Closed Questioning Condition reported significantly more verifiable details than liars in the Preliminary Open Questioning Condition, $\mathrm{t}(20.38)=2.92, p=.008, d=1.00,95 \%$ CI $[.26,1.68]$.To examine how strongly our data supported the hypothesis, we also calculated a Bayes Factor (BF) score (e.g. Wagenmakers et al., 2016). Bayesian analysis showed our data were more in support of the alternative hypothesis than null hypothesis $\left(B F_{01}=5.59\right)$. These results support Hypothesis 1 . In contrast, no difference emerged between truth tellers in the preliminary closed and open questioning conditions for reported verifiable detail, $\mathrm{t}(31)=0.46, p=.651, d=.16,95 \%$ CI [- 
$.53, .84]$. Bayesian analysis showed our data were more in support of the null hypothesis $\left(B F_{10}=0.36\right)$ than alternative hypothesis. These results support Hypothesis 2.

\section{Proportion of verifiable details in the final statement.}

A 2 (Veracity) x 2 (Preliminary Questioning Style) ANOVA with the proportion of verifiable details in the final statement as the dependent variable revealed a significant main effect for Veracity, $F(1,63)=22.004, \mathrm{MSE}=.583, p<0.001, d=1.13,95 \%$ CI $[.60,1.63]$. The main effect for Preliminary Questioning Style, $F(1,63)=2.313$, MSE $=.061, p=0.133$, $d=.50,95 \%$ CI $[.01, .98]$ and the Veracity x Preliminary Questioning Style interaction effect were not significant, $F(1,63)=3.281, \mathrm{MSE}=.087, p=0.074, \eta_{\mathrm{p}}=.05$.

Again, the interaction statistics $(p=.074)$ refers to any type of interaction. The more informative test of Hypothesis 1 is to statistically test for significant differences between the two Preliminary Questioning Style conditions for truth-tellers and liars separately (e.g. Nahari \& Ben-Shakhar, 2011). Thus, we examined the difference in the proportion of verifiable detail reported by truth tellers and liars, for the two preliminary interview style conditions separately, as this addresses Hypothesis 1 and 2 directly.

As Table 3 also shows, liars in the preliminary closed condition reported a significantly greater proportion of verifiable detail in their final statement than liars in the preliminary open condition, $t(32)=2.382, p=0.023, d=.87,95 \% \mathrm{CI}[0.14,1.54]$. Bayesian analysis showed our data were more in support of the alternative hypothesis than the null hypothesis $\left(B F_{10}=2.63\right)$. These results support Hypothesis 1 . In contrast, truth tellers in the preliminary closed questioning Condition reported a similar proportion of verifiable detail in their final statement as truth tellers in the preliminary open condition, $t(31)=.203, p=0.840, d=.06$, $95 \%$ CI $-.62, .74]$. Bayesian analysis showed our data were more in support of the null hypothesis than the alternative hypothesis $\left(B F_{01}=0.34\right)$. These results support Hypothesis 2 . 


\section{Consistency analysis of verifiable sources}

\section{Verifiable Source commissions}

A 2 (Veracity) x 2 (Preliminary Questioning Style) ANOVA was conducted with the number of reported verifiable evidence factor commissions as the dependent variable, and the number of reported verifiable evidence factors in the initial statement as the covariant. This analysis revealed a significant main effect for Veracity, $F(1,62)=4.473$, MSE $=.861, p=$ $0.038, d=.36,95 \%$ CI $[-.13, .83]$. The main effect for Preliminary Questioning Style was not significant, $F(1,62)=0.063, \mathrm{MSE}=.014, p=0.802, d=.10,95 \%$ CI $[-.38, .57]$, but the Veracity x Preliminary Questioning Style interaction effect, $F(1,62)=13.942$, MSE $=2.996$, $p<0.001, \eta_{\mathrm{p}^{2}}=.18$

[Table 4 about here]

Truth tellers in the preliminary open condition reported significantly more Verifiable Source commissions than truth tellers in the preliminary closed condition, $t(23.29)=2.40, p=$ $.025, d=.82,95 \%$ CI $[.08,1.50]$. Bayesian analysis showed our data were more in support of the alternative hypothesis than the null hypothesis $\left(B F_{10}=2.57\right)$. Displaying the opposite pattern, liars in the preliminary closed condition reported significantly more Verifiable Source commissions than liars in the preliminary open condition, $t(16)=2.954, p=.009, d=$ $1.01,95 \%$ CI [.27, 1.69]. Bayesian analysis showed our data were more in support of the alternative hypothesis than null hypothesis $\left(B F_{01}=9.19\right)$. These results support Hypothesis 3 .

\section{Verifiable Source omissions.}

A 2 (Veracity) x 2 (Preliminary Questioning Style) ANOVA was conducted with the number of reported verifiable evidence factor omissions as the dependent variable, and the number of reported verifiable evidence factors in the initial statement as the covariant. This analysis revealed a significant main effect for Veracity, $F(1,62)=13.492, \operatorname{MSE}=5.138, p=$ $0.001, d=.32,95 \%$ CI $[-.17, .80]$ and a main effect for Preliminary Questioning Style, $F(1$, 
$62)=14.695, \mathrm{MSE}=5.596, p<0.001, d=.83,95 \% \mathrm{CI}[.32,1.32]$. Furthermore, the Veracity $\mathrm{x}$ Preliminary Questioning Style interaction effect was significant, $F(1,62)=10.814, \mathrm{MSE}=$ $4.118, p=0.002, \eta_{p^{2}}=.15$.

As Table 4 shows, truth tellers in the preliminary open condition reported significantly more Verifiable Source omissions than truth tellers in the preliminary closed condition, $t(31)=3.57, p=.001, d=1.24,95 \%$ CI $[-.46,1.94]$. Bayesian analysis showed our data were more in support of the alternative hypothesis than null hypothesis $\left(B F_{01}=26.75\right)$. In contrast, no difference emerged for Verifiable Source omissions between liars in the preliminary open condition and liars in the preliminary closed condition, $t(32)=1.44, p=.160, d=.49,95 \% \mathrm{CI}[-$ $.20,1.16]$. Bayesian analysis showed our data were more in support of the null hypothesis than alternative hypothesis $\left(B F_{01}=0.70\right)$.

\section{Verifiable Source repetitions.}

A 2 (Veracity) x 2 (Preliminary Questioning Style) ANOVA was conducted with the number of reported verifiable evidence factor repetitions as the dependent variable, and the number of reported verifiable evidence factors in the initial statement as the covariant. This analysis revealed a significant main effect for Veracity, $F(1,62)=12.598, \operatorname{MSE}=4.683, p=$ $0.001, d=1.52,95 \%$ CI $[.95,2.04]$ and a main effect for Preliminary Questioning Style, $F(1$, $62)=16.702, \operatorname{MSE}=6.209, p<0.001, d=.50,95 \%$ CI $[.01, .98]$. Furthermore, the Veracity $\mathrm{x}$ Preliminary Questioning Style interaction effect was significant, $F(1,62)=12.509, \mathrm{MSE}=$ $4.650, p=0.001, \eta_{\mathrm{p}^{2}}=.17$.

As Table 4 also shows, truth tellers in the preliminary open condition reported significantly more Verifiable Source repetitions than truth tellers in the preliminary closed condition, $t(31)=4.17, p<.001, d=1.45,95 \%$ CI $[.64,2.16]$. Bayesian analysis showed our data were more in support of the null hypothesis than alternative hypothesis $\left(B F_{01}=106.62\right)$. In contrast, no difference emerged for Verifiable Source repetitions between liars in the 
preliminary open condition and liars in the preliminary closed condition, $t(32)=.51, p=.612$, $d=.18,95 \%$ CI $[-.50, .85]$. Bayesian analysis showed our data were more in support of the null hypothesis than alternative hypothesis $\left(B F_{10}=0.33\right)$.

\section{Classificatory accuracy rate based on the final statement}

We ran four discriminant analyses to distinguish between truth tellers and liars in the (i) preliminary probe condition and (ii) preliminary free-recall condition using either the number (or the proportion) of verifiable details reported in the final statement separately. In all cases, the objective group belonging (truthful versus deceptive) was the classifying variable. In the first pair of analysis, the number of verifiable details reported in the final statement was entered as the predictor whereas in the final pair of analysis the proportion of verifiable detail in the final statement was entered as the predictor. We present the cross-validated 'leave-oneout' results. As Table 3 shows, superior classification accuracy was obtained in the preliminary free-recall questioning condition.

\section{[Insert Table 5 around here]}

\section{Receiver Operator Characteristic curve analysis}

To complement the series of discriminant analyses (and to formally test Hypothesis 4), we also conducted a series of four Receiver Operator Characteristic (ROC) analysis to distinguish between truth tellers and liars in the (i) preliminary closed and (ii) preliminary open question conditions, using either the number (or the proportion) of verifiable details reported in the final statement separately. For both variables, the state variable (i.e. the particular dichotomous outcome a positive test - i.e. a higher score - indicates) was truth. Unlike a discriminate analysis, the Area Under the Curve (AUC) of a ROC curve provides a measure of the diagnosticity of the criterion as a whole (with 1- specificity, i.e. false positive rate, plotted on the $\mathrm{x}$-axis and sensitivity, i.e. true positive rate, plotted on the y-axis). 
As Figures 1a-2b indicate, the area under the curve (AUC) obtained for each cue was greater in the preliminary free-recall condition for verifiable detail; .93 for the proportion of verifiable detail) than in the preliminary probe condition (.73 for verifiable detail; .72 for the proportion of verifiable detail).

To formally establish if the AUC's obtained for the number of verifiable details and the proportion of verifiable details reported in the final statement differed between the preliminary open and closed questioning conditions, we conducted two independent AUC comparison tests examining the number and proportion of reported verifiable details separately. For the number of reported verifiable details, the AUC was significantly higher in the preliminary open $(\mathrm{AUC}=.97)$ than in the closed questioning condition $(\mathrm{AUC}=.73), p=$ 0.004. For the proportion of verifiable details reported, the AUC was significantly higher in the preliminary open $(\mathrm{AUC}=.93)$ than in the closed questioning condition $(\mathrm{AUC}=.72), p=$ 0.012. These results indicate superior diagnosticity for both variables in the preliminary open (versus closed) questioning condition, which supports Hypothesis 4.

\section{Discussion}

Study 1 showed that the performance of the Verifiability Approach (VA) is sensitive to the type of preliminary questioning used. When open preliminary questioning was used, liars during the subsequent VA reported final statements containing lower proportion (and overall number) of verifiable details, compared to when preliminary closed questioning was employed (supporting Hypothesis 1). In contrast, the proportion (and overall number) of reported verifiable detail in the final statement did not vary for truth tellers as a function of preliminary questioning type (supporting Hypothesis 2). Furthermore, liars in the preliminary open questioning condition reported final statements containing significantly fewer verifiable source commissions compared to liars in the preliminary closed questioning condition (supporting Hypothesis 3). In contrast, the opposite pattern emerged from honest 
interviewees: truth tellers in the preliminary open questioning condition reported final statements containing significantly more verifiable source commissions compared to truth tellers in the preliminary closed questioning condition. These patterns of behaviour resulted in greater veracity-based verbal differences, and in turn superior classification of liars and truth tellers by the VA, in the preliminary open questioning condition, supporting Hypothesis 4.

Collectively, the above findings make good theoretical sense. Initial open questioning elicits more detailed statements compared to initial closed questioning. This elicitation of addition information has different consequences for liars and truth tellers, due to their adoption of specific verbal strategies. Regardless of initial question type, truth tellers can adopt forthcoming verbal strategies (Hartwig et al., 2007; 2010) and disclose new verifiable detail (Nahari et al., 2014a; 2014b), i.e. reminiscence (Gilbert \& Fisher, 2006; Payne, 1987). In contrast, liars face at least two strategic problems following initial open questioning. First, liars are motivated to withhold verifiable details because they jeopardise their credibility (Nahari et al., 2014a). Second, the more detailed a liar's initial statement is, the progressively more difficult it is to then revise subsequent statements and disclose new verifiable detail in response to the VA's information protocol (IP) without displaying between-statements inconsistency (see Vredeveldt, van Koppen and Granhag, 2017). Interestingly, maintaining between-statement consistency (a strategy favoured by liars; Granhag et al., 1999, 2001, 2003) is an ineffective counter-strategy to the VA if liars do not initially provide checkable information (Nahari et al., 2014a). Thus, eliciting detailed initial statements from liars via initial open questioning appears to supresses their ability to revise their statements in response to the IP and provide new verifiable detail and/or introduced new sources of verifiable information in their subsequent statements.

Although the findings are theoretically interesting, Study 1 suffers from practical 
shortcomings we corrected in Study 2. The objectives of Study 2 are three-fold. First, the automated interviewing procedure used in Study 1 maybe of especially relevant to some settings (i.e. high-traffic, low resource portal crossings where automation of veracity assessment is an advantage due to time pressure), but might considered of low ecological validity and relevance to tradition face-to-face police suspect interviewing settings. Thus, we aimed to replicate the findings of Study 1 using a human interviewer. Second, from Study 1's data, it cannot be known if a) the initial open questioning or b) the subsequent VA procedure was the direct cause of the verbal differences between liars and truth tellers in the open questioning condition. Knowing the direct cause of the effect is critical for the development of optimized interviewing protocols for lie detection via the VA. Thus, in Study 2 we extended Study 1 by manipulating providing (or withholding) the VA's Information Protocol (IP) between two free-recall recall attempts. Third, due to the use of initial closed questioning for half the participants in Study 1, a detailed fine-grained analysis of between-statement consistency (i.e. analysis of omissions, commissions, repetitions and contradictions) was not possible for verifiable detail itself. In contrast, Study 2 examined between-statement consistency for reported verifiable detail in a within-subject settings.

Based upon previous research (Nahari et al., 2014b; Harvey et al., 2017) it is plausible that following an initial questioning, administering an IP instruction will elicit additional verifiable details from truth tellers but not liars in subsequent questioning. As such, we predict that truth tellers in the IP condition will report significantly more verifiable details (and a higher proportion of verifiable details) during their final statement than truth tellers in the control condition (where no IP is administered between two recall attempts) during their final statement. However, no equivalent difference is expected for liars (see Nahari et al., 2014b) (Hypothesis 5). We further predict that truth tellers will report more new verifiable details during their final statement in the IP condition than truth tellers in the control 
condition where no IP was provided, whereas no difference is expected for liars between interviewing conditions (Hypothesis 6). Finally, based upon these hypothesised patterns of results, we predict more accurate classification of suspects by the VA (during the final statement) in the IP condition compared to the Control condition, or using the initial statement (Hypothesis 7).

\section{Study 2}

\section{Method}

\section{Design}

The study used a 2 (Veracity: lie vs. truth) x 2 (Questioning Condition: IP vs. Control) x2 (Statement: initial vs. final) mixed factor experimental design. Veracity and Question Condition were between-subjects' factors and Statement was the within-subjects' factor. The dependent measures were i) the proportion of verifiable detail reported per statement, and ii) the proportion of verifiable reminiscences detail reported.

\section{Ethics}

A favourable ethical review decision was given, prior to the research, by the Science Faculty Ethics Committee (SFEC) and CREST Security Research Ethics Committee (SREC). All participants' rights were protected during the study.

\section{Participants}

Based upon the results of Study 1, a power analysis using G*Power, assuming a medium effect size of $f=0.30(\alpha=0.05)$ for four groups, indicated a sample size of 90 (approx. 23 per cell) would be sufficient for a power of 0.80 . One participant was excluded for not following experimental instructions, leaving a total of 96 volunteers, comprising of 62 females and 34 males, aged between 18 and 52 years $(M=24.06$ years, $S D=7.03,95 \% \mathrm{CI}$ $[22.68,25.51])$, from the University's undergraduate $(n=78)$, postgraduate $(n=14)$ and staff $(n=4)$ communities participated in the study. 


\section{Procedure}

Recruitment protocol, veracity-based instructions and experimental procedure were identical to that of Study 1, with the following exception. Upon indicating they were ready to be interviewed, all honest and dishonest participants (henceforth suspects) were informed that the interview itself would be carried out by a human interviewer. Truth tellers and liars were told that their objective was to convince an investigator (who would read their statement) that they were not responsible for the theft. If the investigator believed they were being truthful, they would - in addition to receiving $£ 10$ for taking part - be entered into a prize-draw for a chance to win one of three additional prizes $(£ 75, £ 100$ or $£ 150)$. However, if the investigator did not believe they were being honest, they would not be entered into the prize draw and would instead be required to write a time-consuming essay about their activities. In actuality, all participants were entered into a prize draw and none were required to write the timeconsuming essay.

Follow this, all remaining instructions were identical to Study 1 and, upon indicating they were prepared to be interviewed, all suspects completed a pre-interview questionnaire. The participants were asked for their demographic information (age, gender, occupation) and to rate their preparation for the interview (on 7-point Likert scales, ranging from 1 (very poor) to 7 (very good)). Upon completion, participants were interviewed.

\section{Interview protocol}

All interviews (conducted by an interviewer blind to the veracity condition) were audio recorded and began identically. Participants were welcomed, asked to take a seat, and were told why the interview was occurring (they were a suspect). The interviewer then explained there was just one question and thus the interviewee should provide as much information as possible. The interviewer then asked them to describe, in as much detail as possible, their activities and whereabouts for the 30-minute time period. After they had responded, the 
interviewer thanked the participant for their time, but requested to wait in the interview room for the interviewer to return. The interviewer left the room for 2 minutes before returning.

At this stage, half of the truth tellers and liars were randomly allocated to either the IP condition or the control condition (with no IP). In the IP condition ( $n=48 ; 24$ truth tellers and 24 liars). The interviewer returned and explained he needed to clarify some of the information initially provided and so would be asking the interviewee again to describe his/her activities over the 30-minute timeframe. First however, the same IP instruction used in Study 1 was read verbatim by the interviewer from a script (Appendix 2). The interview then progressed to the final question. In the control condition $(n=48 ; 24$ truth tellers and 24 liars), the interviewer returned and explained he needed to clarify some of the information initially provided and so would be asking the interviewee again to describe their activities over the 30-minute timeframe. No IP instruction was used and so the interviewer then progressed to the final question.

All participants $(n=96)$ were asked the same final question: to report, in as much detail as possible, their activities and whereabouts for the 30-minute time period. Once finished, the interviewer thanked the participants for their time and the experimenter returned.

After the interview, the participants completed the same post-interview questionnaire used in Study 1, with one additional question. To serve as a manipulation check, all participants were asked to report on a 7-point scale (ranging from 1 [extremely unlikely] to 7 [extremely likely] the extent they believed that the verifiable detail they reported in their statements would be used by the analysts to make credibility judgements. Once completed, participants were debriefed as in Study 1 and awarded $£ 10$ honorarium. All participants were entered into the prize draw to win one of the three additional cash prizes.

\section{Coding}

We coded statements for: i) the number of reported verifiable and unverifiable details; 
ii) the consistency (omissions, repetitions, commissions and contradictions) of reported information; iii) the consistency of verifiable sources reported per statement. As with Study 1, we additionally calculated the proportion of verifiable detail per statement. Proportions were calculated by dividing the number of reported verifiable details in each statement by the total number of reported details in each statement: (proportion of verifiable detail $=$ number of verifiable details/ [number of verifiable details + number of unverifiable details]).

\section{Coding the verifiability of statements and inter-rater reliability.}

Coding was identical to Study 1 and the same coder was used in both studies. All statements were first rated by one coder (blind to the experimental conditions). A second coder (also blind to the veracity of the statements) coded a random selection of 24 statements (25\%). The ICC was good and therefore satisfactory for total detail $[\mathrm{ICC}]=.80,95 \% \mathrm{CI}[.52$, $.92](p<0.001)$, verifiable detail [ICC $]=.85,95 \%$ CI $[.64, .94](p<0.001)$, unverifiable detail $[\mathrm{ICC}]=.78,95 \% \mathrm{CI}[.49, .91](p<0.001)$

\section{Coding the consistency of reported detail}

All reported detail (verifiable and unverifiable) in both statements was then coded for consistency as an i) omission; ii) commission; iii) repetition or iv) contradiction (see Fisher et al., 2013; Vredeveldt et al., 2014). However, contradictions did not occur in our data, so only analysis of omissions, commission and repetitions are presented. All statements were first rated by one coder (blind to the experimental conditions). A second coder (also blind to the veracity of the statements) coded a random selection of 24 statements $(25 \%)$. The ICC was good and therefore satisfactory for commissions $[\mathrm{ICC}]=.77,95 \% \mathrm{CI}[.46, .90](p<0.001)$, repetitions $[\mathrm{ICC}]=.85,95 \% \mathrm{CI}[.65, .93](p<0.001)$ and omissions $[\mathrm{ICC}]=.79,95 \% \mathrm{CI}$ $[.51,9](p<0.001)$.

\section{Coding the verifiability source consistency.}

Following study 1 , we distinguished between six sources that contribute to the potential 
verifiability of a suspect's statement. A participant's verifiable source score could range from 0 (no verifiable sources) to 6 (all verifiable sources) reported per statement. A second coder was randomly allocated the statements given by a sample of $24(25 \%)$ participants and scored them for the occurrence of verifiable sources. Inter-rater reliabilities between the two coders for the verifiable factor score were measured via intra-class correlation coefficients (ICC). The ICC was good and therefore satisfactory for verifiable source score $[\mathrm{ICC}]=.82,95 \% \mathrm{CI}$ $[.58, .92](p<0.001)$

Each verifiable source was classified as either omitted (present in statement 1, absent in statement two); committed (present in statement 2, absent in statement 1) or repeated (present in both statements and corresponding (see Fisher et al., 2013; Vredeveldt et al., 2014). A second coder was randomly allocated the statements given by a sample of $24(25 \%)$ participants and scored them for the occurrence of verifiable sources. Inter-rater reliabilities between the two coders for the verifiable factor score were measured via intra-class correlation coefficients (ICC). The ICC was good and therefore satisfactory for verifiable source commissions $[\mathrm{ICC}]=.92,95 \% \mathrm{CI}[.81, .96](p<0.001)$, verifiable source omissions $[\mathrm{ICC}]=.71,95 \% \mathrm{CI}[.32, .87](p=0.002)$, and verifiable source repetitions $[\mathrm{ICC}]=.82,95 \%$ CI $[.59, .92](p<0.001)$.

\section{Supplementary analysis section}

Below are presented the results for the number, proportion and consistency (number of commissions, omissions and repetitions) of reported verifiable details. For additional analysis of the consistency of reported verifiable sources, together with the analysis of the number and consistency of reported unverifiable detail, see the supplementary analysis section.

\section{Results}

\section{Manipulation Check}




\section{Veracity manipulation check.}

Truthful mock-suspects (henceforth suspects) reported their overall truthfulness rating as high $(M=98.13 \%, S D=6.73 \%, 95 \% \mathrm{CI}[95.96 \%, 99.76 \%])$ and deceptive mock-suspects reported a significantly lower truthfulness rating $(M=26.67 \%, S D=26.76 \%, 95 \% \mathrm{CI}$ $[19.38 \%, 34.70 \%]), t(52.927)=17.939, p<0.001, d=3.66,95 \%$ CI $[2.96,4.25]$. This supports the validity of the veracity manipulation.

\section{IP manipulation check.}

Mock suspects in the IP-present condition ( $M=6.25$ on a 7 -point Likert scale, ranging from 1 [extremely unlikely] to 7 [extremely likely], $S D=1.12 .95 \% \mathrm{CI}[5.91,6.53])$ reported believing that their statements verifiable detail would be used by the analysts to make credibility judgements significantly more than mock-suspects in the control (IP-absent) condition $(M=4.42, S D=1.58,95 \%$ CI $[3.93,4.88]), t(84.66)=6.55, p<0.001, d=1.3495 \%$ CI $[.87,1.76]$. This supports the validity of the IP manipulation.

\section{Self-reported motivation levels}

\section{Motivation to be convincing.}

The reported motivation of suspects was relatively high $(M=6.08, S D=.88,95 \% \mathrm{CI}$ [5.90, 6.25]) on a 7-point Likert scale. A 2 (Veracity: Lie vs. Truth) X 2 (Questioning Condition: IP vs. Control) analysis of variance (ANOVA) showed no main effect for Veracity or Questioning Condition, and no Veracity X Questioning Condition interaction, all $F^{\prime} \mathrm{s}<2.49$, all $p$ 's $>.118$.

\section{Hypothesis Testing}

\section{Number of verifiable details}

A 2 (Veracity: lie/ truth) x 2 (Questioning Condition: Information Protocol/ Control) x 2 (Statement: initial/ final) mixed factors ANOVA with Veracity and Questioning Condition as the between-subject factors and Statement as the within-subjects factor was conducted 
using the number of reported verifiable details as the dependent variable. This analysis revealed significant main effects for Veracity, $F(1,92)=20.54, \mathrm{MSE}=7512.51, p<.001, d=$ $.85,95 \%$ CI $[.42,1.25]$; Questioning Condition, $F(1,92)=5.78, \mathrm{MSE}=2113.38, p=.018$, $d=.36,95 \%$ CI $[-.04, .76]$; and Statement, $F(1,92)=37.99, \mathrm{MSE}=1255.63, p<.001, d=.32$, $95 \%$ CI $[.03, .60]$. In addition, the Statement $x$ Veracity interaction, $F(1,92)=13.72$, MSE $=$ 453.26, $p<.001$, partial eta ${ }^{2}=.13$; the Statement $\mathrm{x}$ Questioning condition interaction, $F(1,92)=11.92, \mathrm{MSE}=393.88, p=.001$, partial $\mathrm{eta}^{2}=.12$ and the the three-way Statement $\mathrm{x}$ Questioning condition $\mathrm{x}$ Veracity interaction were all significant, $F(1,92)=24.09$, MSE= $796.26, p<.001$, partial eta ${ }^{2}=.21$. Since the three-way interaction is the most informative of all the significant effects, only the three-way interaction is discussed.

[Table 6 about here]

Using only the initial statements, no significant difference in reported verifiable detail emerged between truth tellers in the control condition and truth tellers in the IP Condition, $t(46)=.559, p=0.147, d=.42,95 \%$ CI $[-.16, .98]$. Bayesian analysis showed our data were more in support of the null hypothesis than alternative hypothesis $\left(B F_{10}=0.69\right)$. These results are not surprising as the IP was only introduced after the initial statement. No significant difference in reported verifiable detail emerged between liars in the control versus liars in the IP Condition, $t(46)=.56, p=.579, d=.16,95 \%$ CI [-.41, .79]. Bayesian analysis showed our data were more in support of the null hypothesis than alternative hypothesis $\left(B F_{10}=0.33\right)$

Using only the second statements, truth tellers in the IP condition reported significantly more verifiable detail than truth tellers in the control condition, $t(39.56)=4.001, p<0.001, d$ $=1.15,95 \%$ CI $[.53,1.75]$. Bayesian analysis showed our data were more in support of the alternative hypothesis than null hypothesis $\left(B F_{10}=108.40\right)$. In contrast, no significant difference in terms of reported verifiable detail emerged between liars in the control 
condition and liars in the IP condition, $t(46)=.171, p=.865, d=.05,95 \%$ CI $[-.52, .61]$. Bayesian analysis showed our data were more in support of the null hypothesis than alternative condition $\left(B F_{10}=0.34\right)$. These results support Hypothesis 5.

\section{Proportion of verifiable detail}

A 2 (Veracity: lie/ truth) x 2 (Questioning Condition: Information Protocol/ Control) x 2 (Statement: initial/ final) mixed factors ANOVA with Veracity and Questioning Condition as the between-subject factors and Statement as the within-subjects factor was conducted using the proportion of verifiable detail reported as the dependent variable. The ANOVA revealed main effects for Veracity, $F(1,92)=4.165, \mathrm{MSE}=.300, p=.044, d=.34,95 \% \mathrm{CI}$ $[-.07, .74]$, Questioning Condition, $F(1,92)=4.191, \mathrm{MSE}=.302, p=.043, d=.34,95 \% \mathrm{CI}[-$ $.07, .74]$ and Statement, $F(1,92)=20.948, \mathrm{MSE}=.199, p<.001, d=.33,95 \% \mathrm{CI}[-.08, .73]$.

The Statement $\mathrm{x}$ Questioning Condition interaction effect was significant, $F(1,92)=$ 4.957, MSE $=.047, p=.028, \eta p^{2}=.05$, but the Statement $\mathrm{x}$ Veracity interaction effect, $F(1,92)=1.621, \mathrm{MSE}=.015, p=.206, \eta p^{2}=.02$ and the Veracity $\mathrm{x}$ IP interaction effect were not, $F(1,92)=.937, \mathrm{MSE}=.067, p=.336, \eta p^{2}=.01$. However, the three-way Statement $\mathrm{x}$ Veracity x Questioning Condition interaction was also significant, $F(1,92)=4.072$, MSE = $.039, p=.047, \eta p^{2}=.04$. Since the three-way interaction is the most informative effect of all significant effects, only the three-way interaction is discussed.

Using only the initial statements, no differences emerged between truth tellers in the control condition and truth tellers in the IP condition, $t(46)=1.226, p=.227, d=.37,95 \% \mathrm{CI}$ $[-.20, .94]$. Bayesian analysis showed our data were more in support of the null hypothesis than alternative hypothesis $\left(B F_{10}=0.53\right)$. This null effect is not surprising as the IP was only introduced after the initial statement. Furthermore, using only the final statements, no differences emerged between liars in the control condition and liars in the IP condition, $t(46)$ 
$=.657, p=.514, d=.19,95 \% \mathrm{CI}[-.38, .75]$. Bayesian analysis showed our data were more in support of the null hypothesis than alternative hypothesis $\left(B F_{10}=0.34\right)$.

Using only the final statements, truth tellers in the IP condition, $t(46)=3.379, p=.001$, $d=.94,95 \% \mathrm{CI}[-.30, .1 .52]$ reported statements with a significantly higher proportion of verifiable detail than truth tellers in the control condition. Bayesian analysis showed our data were more in support of the alternative hypothesis than null hypothesis $\left(B F_{10}=22.40\right)$. In contrast, using only the final statements, no differences emerged between liars in the control condition and liars in the IP condition, $t(46)=.621, p=.538, d=.18,95 \%$ CI $[-.39, .74]$. Bayesian analysis showed our data were more in support of the null hypothesis than alternative hypothesis $\left(B F_{10}=0.34\right)$. These results support Hypothesis 5 .

\section{Consistency analysis of reported verifiable details}

\section{Verifiable commissions}

A 2 (Veracity: lie/ truth) x 2 (Questioning Condition: Information Protocol/ Control) between-subjects ANOVA was conducted using the number of verifiable commissions as the dependent variable, and with the number of verifiable details reported in statement 1 as the covariant. The ANOVA revealed a main effect for Veracity, $F(1,91)=8.55, \mathrm{MSE}=433.45$, $p=.004, d=.47,95 \%$ CI $[.06,0.87]$. A main effect also emerged for Questioning Condition, $F(1,91)=15.61, \mathrm{MSE}=790.99, p<.001, d=.60,95 \%$ CI $[.18,1.00]$. Furthermore, the Veracity $\mathrm{x}$ Questioning condition interaction was significant, $F(1,91)=25.68$, MSE $=$ 1301.68, $p<.001$, partial eta ${ }^{2}=.22$.

\section{[Table 7 about here]}

Truth tellers in the IP condition $(M=18.96, S D=11.49)$ reported more verifiable commissions than truth tellers in the control condition $(M=3.92, S D=5.31), t(46)=5.82, p$ $<0.00, d=1.68,95 \%$ CI $[1.00,2.31]$. Bayesian analysis showed our data were more in support of the alternative hypothesis than null hypothesis $\left(B F_{10}=22671.00\right)$. No difference 
emerged between liars in the IP $(M=3.38, S D=4.85)$ and control conditions $(M=4.42, S D$ $=8.74)$ for reported verifiable commissions, $t(46)=.511, p=0.612, d=.15,95 \%$ CI [-.42, .71]. Bayesian analysis showed our data were more in support of the null hypothesis $\left(B F_{10}=\right.$ $0.32)$.

\section{Verifiable omissions}

A 2 (Veracity: lie/ truth) x 2 (Questioning Condition: Information Protocol/ Control) between-subject ANOVA was conducted using the number of verifiable omissions as the dependent variable, and with the number of verifiable details reported in statement 1 as the covariant. No significant main effects emerged, all $F$ 's $<.75$, all $p$ 's $>.390$.

\section{Verifiable repetitions}

A 2 (Veracity: lie/ truth) x 2 (Questioning Condition: Information Protocol/ Control) between-subject ANOVA was conducted using the number of verifiable repetitions as the dependent variable, and with the number of verifiable details reported in statement 1 as the covariant. No significant main effects emerged, all $F^{\prime}$ s $<.75$, all p's $>.390$.

\section{Classificatory accuracy}

We ran eight discriminant analyses to distinguish between truth tellers and liars in the (i) Information Protocol condition and (ii) and control condition separately, using either the number (or the proportion) of verifiable details reported in statement 1 and statement 2 separately. In all cases, the objective group belonging (truthful versus deceptive) was the classifying variable. In the four analyses, the number of verifiable details reported in the final statement was entered as the predictor whereas in the final four analyses the proportion of verifiable detail in the final statement was entered as the predictor. We present the crossvalidated 'leave-one-out' results. As Table 7 shows, optimal classification accuracy was obtained in the IP condition using statement 2 (supporting Hypothesis 7). 


\section{Receiver Operator Characteristic curve analysis}

To complement the series of discriminant analyses (and to formally test Hypothesis 7), we also conducted four Receiver Operator Characteristic (ROC) analyses to distinguish between truth tellers and liars in the (i) IP condition and (ii) control conditions separately, using either the number (or the proportion) of verifiable details reported in statement 2 only. For both variables, the state variable (i.e. the particular dichotomous outcome a positive test i.e. a higher score - indicates) was truth. Unlike a discriminate analysis, the Area Under the Curve (AUC) of a ROC curve provides a measure of the diagnosticity of the criterion as a whole (with 1- specificity, i.e. false positive rate, plotted on the $\mathrm{x}$-axis and sensitivity, i.e. true positive rate, plotted on the y-axis).

[Insert Figures 3a-4b around here]

As Figures 3a-4b indicate, the area under the curve (AUC) obtained for each cue was greater in the IP condition $(\mathrm{AUC}=.91$ for the number of verifiable detail; $\mathrm{AUC}=.73$ for the proportion of verifiable details; ) than in the control condition (AUC $=.77$ for the number of verifiable details; $\mathrm{AUC}=.63$ for proportion of verifiable details).

To formally establish if the AUC's obtained for the number of verifiable details and the proportion of verifiable details reported differed between the IP and control conditions, we conducted two independent AUC comparison tests. For the number of reported verifiable details reported, the difference between AUC in the IP $(\mathrm{AUC}=.91)$ and control conditions $(\mathrm{AUC}=.77)$ was significant, $p=0.047$. However, for the proportion of verifiable details reported, the difference between AUC in IP $(\mathrm{AUC}=.73)$ and control conditions $(\mathrm{AUC}=.63)$ was not significant, $p=0.177$. These results indicate superior diagnosticity for the number of verifiable details reported in the Information.

\section{Discussion}

Providing (versus withholding) the information protocol (IP) between recall attempts 
resulted in truth tellers, but not liars, reporting statements containing more verifiable information - including reporting more new verifiable sources - during subsequent questioning (supporting Hypothesis 5). Providing the IP also magnified the elicitation of new, previously unreported verifiable details from truth tellers (a measure of an interviewees' between-statement consistency; more new detail indicating less consistency). In contrast, liars did not revise their statements, and their statements did not vary in the amount or proportion of verifiable detail reported, nor in terms of new verifiable details reported or new verifiable sources reported in the second free recall across both conditions (indicating similar levels of between-statement consistency), supporting Hypothesis 6. This pattern of results is consistent with the verbal strategy literature. Liars are motivated to not only avoid disclosing verifiable information (Nahari et al., 2014a) but also to maintain between-statement consistency by repeating previously disclosed details (e.g. Granhag et al., 1999). In contrast, truth tellers can freely report verifiable information they can reconstruct from memory (e.g. Granhag et al., 2001), especially when prompted via the IP instruction (Nahari et al., 2014b). Thus, the IP appears to enhance inconsistence from truth tellers (but not liars) by critically eliciting the reporting of new verifiable information. Accordingly, superior classification rates emerged in the IP (compared to the control) condition, supporting hypothesis 7.

\section{General Discussion}

Whereas previous research has focused upon eliciting more verifiable detail from truth tellers (e.g. Harvey et al., 2017b; Nahari et al., 2014b), Study 1 focused on means of supressing liars' willingness to report verifiable detail by exploiting their motivation to maintain between-statement consistency. Study 1 demonstrated that open-ended preliminary questioning (compared to closed questioning) can actively reduce liar's inclination to report high proportions of verifiable details in subsequent retrieval attempts. This makes good theoretical sense; the more detailed the original statements (and free-recall questioning 
typically elicits more information that closed questioning), the more difficult later revising the statement becomes whist simultaneously maintaining consistency. Truth tellers, in contrast to liars, do not prioritize consistency when repeatedly interviewed. Instead, honest interviewees can openly disclose information they can reconstruct from memories (Granhag et al., 1999, 2001, 2003). This resulted in the proportion of verifiable detail reported during the VA being most diagnostic following initial open questioning. The results suggest that initiating interviews with free-recall type of questions - recommended by best-practice guideline and empirical research for obtaining complete and accurate accounts from witnesses - is beneficial for lie detection using the VA framework. As such, the VA can be readily integrated within accepted interviewing practice.

Study 2 aimed to isolate the effect of the VA's IP component on the proportion and consistency of verifiable detail elicited during subsequent interviewing. Study 2 demonstrated that the IP actively encouraged truth tellers (but not liars) to disclose additional new, previously unreported verifiable detail in within-subject settings. This finding is consistent with research employing the IP in between-subject designs (e.g. Harvey et al., 2017a; Nahari et al., 2014b). Again, these findings make good sense; liars prioritize between-statement consistency (Granhag et al., 1999, 2001, 2003) and resist disclosing new verifiable detail (even in response to the IP; Nahari et al., 2014b), whereas when prompted truth tellers are willing to report new checkable information. Collectively, both studies suggest the VA framework can be usefully extended to elicit consistency-based cues to deception.

We cannot exclude the possibility that combinations of open and closed preliminary questions would lead to different results. Specifically, combining both question types in preliminary question may be advantageous (e.g. Hartwig et al., 2011), as individuals report more information with increased retrieval attempts (Colwell, Hiscock-Anisman, \& Fede, 2013; Gilbert et al., 2006; Payne, 1987). Indeed, if the arguments advanced previously are 
correct, then theoretically any questioning technique that elicits a detailed initial statement should have an enhancing effect on the subsequent VA procedure.

It could be argued the methods employed in both our studies - although consistent with the previous VA literature - do not secure the ground-truth (the objective veracity) of truth teller's statements. However, failure to explore self-generated narratives with ground truth, is a limitation of the wider deception literature. Research into self-generated narratives may reveal cues to deception not present in other more tightly controlled (and less realistic) scenarios (Vrij et al., 2017). It is also noteworthy that truth tellers overwhelmingly reported disclosing truthful information in both Study 1, (>97\% truthful) and Study $2(>98 \%$ truthful). We have no reason to distrust these self-reported percentages.

In terms of future research, one interesting direction for future research is the integration of automated fact-checking (Hassan, Arslan, Li \& Tremayne, 2016; Hassan et al., 2017) and verifiability approach (Nahari et al., 2014a, 2014b) literatures. Study 1 indicated that automated interviewing may be employed to elicit statements from deceptive and truthful suspects that differ in terms of verifiable detail reported (also see Harvey et al., 2017c). Previous research has demonstrated the utility of automatic statement coding for information (e.g. Kleinberg et al. 2017; 2018). Furthermore, automatic 'fact-checking' protocols have been explored verifying a diversity of information, including web-based claims (Karadzhov, Nakov, Marquez, Barron-Cedeno \& Koychev, 2017) simple statistical claims (Vlachos \& Riedel, 2015) and journalistic claims (Vlachos \& Riedel, 2014). Future research could explore end-to-end automated verbal credibility assessment, combining automated interviewing, coding and statement verification as a means of effective statement verification in time-pressured, resource-limited settings. Interestingly, notifying truthful and deceptive interviewees that such a machine-based fact-checking protocol will be employed may influence their respective strategy selection (e.g. Orthey, Vrij, Leal, \& Blank, 2017), effecting 
their verbal behaviour and the diagnostic utility of the VA (e.g. Nahari et al., 2014b; Harvey et al., 2017d). Future research should explore this possibility.

In conclusion, preliminary question style affected the verbal behaviour of liars and truth tellers differently during a subsequent VA procedure. Initial open (compared to the closed) questioning suppressed the subsequent reporting of verifiable information by liars, thus leading to superior discrimination between suspects via the VA (Study 1). Providing the IP (versus a control with no IP) elicited final statements containing more verifiable detail, a greater proportion of verifiable detail, and more newly reported verifiable detail (and verifiable sources) from truth tellers but not liars in their final statement (Study 2). Thus, theory-driven interviewing techniques designed to exploit liar's preferred counter-interview strategies may provide a useful means of refining the VA. 


\section{References}

Bell, B. E., \& Loftus, E. F. (1988). Degree of detail of eyewitness testimony and mock juror judgments. Journal of Applied Social Psychology, 18, 1171-1192. doi:10.1037/00223514.56.5.669

Bond, C. F., \& DePaulo, B. M. (2006). Accuracy of deception judgments. Personality and social psychology Review, 10(3), 214-234. https://doi.org/10.1207/s15327957pspr1003_2

Centrex (2004) Practical Guide to Investigative Interviewing. London: Central Police Training and Development Authority.

Clarke, C., Milne, R., \& Bull, R. (2011). Interviewing suspects of crime: The impact of PEACE training, supervision and the presence of a legal advisor. Journal of Investigative Psychology and Offender Profiling, 8(2), 149-162, DOI: 10.1002/jip.144

Cohen, J. (1992). A power primer. Psychological bulletin, 112(1), 155-159.

Colwell, K., Fede, J., \& Hiscock-Anisman, C. (2013). Assessment Criteria Indicative of Deception: an example of the new paradigm of differential recall enhancement. In B.S. Cooper et al. (Eds.) Applied Issues in Investigative Interviewing, Eyewitness Memory, and Credibility Assessments. New York: Springer Science and Business Media.

Davies, G. M., Westcott, H. L. and Horan, N. (2000) e impact of questioning style on the content of investigative interviews with suspected child sexual abuse victims. Psychology, Crime and Law 6: 81-97, http://dx.doi.org/10.1080/10683160008410834

DePaulo, B. M., Lindsay, J. J., Malone, B. E., Muhlenbruck, L., Charlton, K., \& Cooper, H. 
(2003). Cues to deception. Psychological Bulletin, 129(1), 74. doi:10.1037/00332909.129.1.74

Faul, F., Erdfelder, E., Lang, A. G., \& Buchner, A. (2007). G* Power 3: A flexible statistical power analysis program for the social, behavioral, and biomedical sciences. Behavior research methods, 39(2), 175-191.

Ganis, G., Rosenfeld, J. P., Meixner, J., Kievit, R. A., \& Schendan, H. E. (2011). Lying in the scanner: covert countermeasures disrupt deception detection by functional magnetic resonance imaging. Neuroimage, 55(1), 312-319, http://dx.doi.org/10.1016/j.neuroimage.2010.11.025

Gilbert, J. A., \& Fisher, R. P. (2006). The effects of varied retrieval cues on reminiscence in eyewitness memory. Applied Cognitive Psychology, 20(6), 723-739, doi: 10.1002/acp. 1232

Granhag, P. A., \& Strömwall, L. A. (1999). Repeated interrogations-Stretching the deception detection paradigm. Expert Evidence, 7, 163-174. doi:10.1023/a:1008993326434.

Granhag, P. A., \& Strömwall, L. A. (2001). Deception detection based on repeated interrogations. Legal and Criminological Psychology, 6, 85-101. doi:10.1348/135532501168217.

Granhag, P. A., Strömwall, L. A., \& Jonsson, A.C. (2003). Partners in crime: How liars in collusion betray themselves. Journal of Applied Social Psychology, 33, 848-868. doi:10.1111/j.1559- 1816.2003.tb01928.x.

Hartwig, M., \& Bond Jr, C. F. (2011). Why do lie-catchers fail? A lens model meta-analysis 
of human lie judgments. Psychological bulletin, 137(4), 643. doi:10.1037/a0023589

Hartwig, M., Granhag, P. A., \& Strömwall, L. A. (2007). Guilty and innocent suspects' strategies during police interrogations. Psychology, Crime \& Law, 13(2), 213-227. doi:10.1080/10683160600750264

Hartwig, M., Granhag, P. A., Stromwall, L. A., \& Doering, N. (2010). Impression and information management: On the strategic self-regulation of innocent and guilty suspects. Open Criminology Journal, 3, 10-16. doi:10.2174/1874917801003020010

Hartwig, M., Granhag, P. A., Strömwall, L. A., \& Kronkvist, O. (2006). Strategic use of evidence during police interviews: When training to detect deception works. Law and human behavior, 30(5), 603-619, doi: 10.1007/s10979-006-9053-9

Hartwig, M., Granhag, P. A., Strömwall, L. A., \& Vrij, A. (2005). Detecting deception via strategic disclosure of evidence. Law and human behavior, 29(4), 469. doi:10.1007/s10979-005-5521-x

Hartwig, M., Granhag, P. A., Stromwall, L., Wolf, A. G., Vrij, A., \& Hjelmsäter, E. R. A. (2011). Detecting deception in suspects: Verbal cues as a function of interview strategy. Psychology, Crime \& Law, 17(7), 643-656, doi.org/10.1080/10683160903446982

Harvey, A. C., Vrij, A., Leal, S., Lafferty, M., \& Nahari, G. (2017b). Insurance based lie detection: Enhancing the verifiability approach with a model statement component. Acta Psychologica, 174, 1-8, http://dx.doi.org/10.1016/j.actpsy.2017.01.001

Harvey, A. C., Vrij, A., Nahari, G., \& Ludwig, K. (2017a). Applying the Verifiability Approach to insurance claims settings: Exploring the effect of the information 
protocol. Legal and Criminological Psychology. doi: 10.1111/1crp.12092

Hassan, N., Tremayne, M., Arslan, F., \& Li, C. (2016). Comparing automated factual claim detection against judgments of journalism organizations. In Computation+ Journalism Symposium.

Hassan, N., Arslan, F., Li, C., \& Tremayne, M. (2017, August). Toward automated factchecking: Detecting check-worthy factual claims by ClaimBuster. In Proceedings of the 23rd ACM SIGKDD International Conference on Knowledge Discovery and Data Mining (pp. 1803-1812). ACM.

Home Office (UK) (2007) Achieving Best Evidence in Criminal Proceedings: guidance on interviewing victims and witnesses, and using special measures. London: HMSO.

Johnson, M. K. (2006). Memory and reality. American Psychologist, 61(8), 760-771, DOI: 10.1037/0003-066X.61.8.760

Karadzhov, G., Nakov, P., Màrquez, L., Barron-Cedeno, A., \& Koychev, I. (2017). Fully automated fact checking using external sources. arXiv preprint arXiv:1710.00341.

Kleinberg, B., Mozes, M., Arntz, A., \& Verschuere, B. (2017). Using Named Entities for Computer-Automated Verbal Deception Detection. Collabra: Psychology, 3(1), 21, doi: http://doi.org/10.1525/collabra.80

Kleinberg, B., Van Der Toolen, Y., Vrij, A., Arntz, A., \& Verschuere, B. (2018). Automated verbal credibility assessment of intentions: The model statement technique and predictive modeling. Applied cognitive psychology, 32(3), 354-366. DOI: 10.1002/acp.3407 
Lamb, M. E., Hershkowitz, I., Sternberg, K. J., Esplin, P. W., Hovath, M., Manor, T. and Yudilevitch, L. (1996) Effects of investigative utterance types on Israeli children's responses. International Journal of Behavioral Development 19(3): 627-637, doi: $\underline{10.1177 / 016502549601900310}$

Leach, A. M., Ammar, N., England, D. N., Remigio, L. M., Kleinberg, B., \& Verschuere, B. J. (2016). Less is more? Detecting lies in veiled witnesses. Law and human behavior, 40(4), 401, http://dx.doi.org/10.1037/1hb0000189

Masip, J., \& Herrero, C. (2013). 'What would you say if you were guilty?' Suspects’ strategies during a hypothetical behavior analysis interview concerning a serious crime. Applied Cognitive Psychology, 27, 60-70. doi:10.1002/acp.2872

Myklebust, T., \& Bjørklund, R. A. (2006). The effect of long-term training on police officers' use of open and closed questions in field investigative interviews of children (FIIC). Journal of Investigative Psychology and Offender Profiling, 3(3), 165-181, doi: $10.1002 /$ jip.52

Nahari, G. (2016). When the long road is the shortcut: A comparison between two coding methods for content-based lie detection tools. Psychology, Crime and Law, 22, 10001014.

Nahari, G. (2017). Verifiability approach: Applications in different judgmental settings. In T. Docan-Morgan (Ed.). The Handbook of Deceptive Communication. Hampshire: Palgrave Macmillan. 
Nahari, G., \& Vrij, A. (2014). Can I borrow your alibi? The applicability of the verifiability approach to the case of an alibi witness. Journal of Applied Research in Memory and Cognition, 3(2), 89-94, http://dx.doi.org/10.1016/j.jarmac.2014.04.005

Nahari, G., \& Vrij, A. (2015). Can someone fabricate verifiable details when planning in advance? It all depends on the crime scenario. Psychology, Crime \& Law, 21, 987999. doi:10.1080/ 1068316X.2015.1077248

Nahari, G., Vrij, A., \& Fisher, R. P. (2012). Does the truth come out in the writing? Scan as a lie detection tool. Law and Human Behavior, 36(1), 68, http://dx.doi.org/10.1037/h0093965

Nahari, G., Vrij, A., \& Fisher, R. P. (2014a). Exploiting liars' verbal strategies by examining the verifiability of details. Legal and Criminological Psychology, 19, 227-239. doi:10.1111/j.2044- 8333.2012.02069.x

Nahari, G., Vrij, A., \& Fisher, R. P. (2014b). The verifiability approach: Countermeasures facilitate its ability to discriminate between truths and lies. Applied Cognitive Psychology, 28, 122-128. doi:10.1002/acp.2974

Orthey, R., Vrij, A., Leal, S., \& Blank, H. (2017). Strategy and misdirection in forced choice memory performance testing in deception detection. Applied Cognitive Psychology, 31(2), 139-145, https://doi.org/10.1002/acp.3310

Oxburgh, G. E., Myklebust, T., \& Grant, T. (2010). The question of question types in police interviews. Int. J. Speech Lang. Law, 17(1), 45-66, doi: 101.1558/ijsll.v17il.45

Payne, D. G. (1987). Hypermnesia and reminiscence in recall: A historical and empirical review. Psychological Bulletin, 101(1), 5, http://dx.doi.org/10.1037/0033- 
2909.101.1.5

Strömwall, L. A., Hartwig, M., \& Granhag, P. A. (2006). To act truthfully: Nonverbal behaviour and strategies during a police interrogation. Psychology, Crime \& Law, 12(2), 207-219. doi: 10.1080/10683160512331331328

Strömwall, L. A., \& Willén, R. M. (2011). Inside criminal minds: Offenders' strategies when lying. Journal of Investigative Psychology and Offender Profiling, 8(3), 271-281, doi: 10.1002/jip.148

Vlachos, A., \& Riedel, S. (2015). Identification and verification of simple claims about statistical properties. In Proceedings of the 2015 Conference on Empirical Methods in Natural Language Processing (pp. 2596-2601). Association for Computational Linguistics.

Vlachos, A., \& Riedel, S. (2014). Fact checking: Task definition and dataset construction. In Proceedings of the ACL 2014 Workshop on Language Technologies and Computational Social Science (pp. 18-22).

Vredeveldt, A., van Koppen, P. J., Granhag, P. A. (2014). The inconsistent suspect: A systematic review of different types of consistency in truth tellers and liars. In R. Bull (Ed.), Investigative Interviewing (pp. 183-207). New York: Springer Science+Business Media. doi: 10.1007/978-1-4614-9642-7_10

Vrij, A. (2008). Detecting lies and deceit: Pitfalls and opportunities. Chichester, UK: John Wiley \& Sons.

Vrij, A. (2015). Verbal Lie Detection tools: Statement validity analysis, reality monitoring and scientific content analysis. In P. A. Granhag, A. Vrij, \& B. Verschuere. 
(Eds.) Detecting Deception Current Challenges and Cognitive Approaches. Chichester: John Wiley \& Sons.

Vrij, A., Fisher, R., Blank, H. (2017). A cognitive approach to lie detection: A meta-analysis. Legal and Criminological Psychology, 22, 1-21. DOI:10.1111/1crp.12088

Vrij, A., \& Granhag, P. A. (2012). Eliciting cues to deception and truth: What matters are the questions asked. Journal of Applied Research in Memory and Cognition, 1, 110-117. doi:10.1016/j.jarmac.2012.02.004

Vrij, A., \& Granhag, P. A. (2014). Eliciting information and detecting lies in intelligence interviewing: An overview of recent research. Applied Cognitive Psychology, 28, 936-944. doi:10.1002/ acp.3071

Vrij, A., Mann, S., Kristen, S., \& Fisher, R. P. (2007). Cues to deception and ability to detect lies as a function of police interview styles. Law and human behavior, 31(5), 499-518. doi: 10.1007/s10979-006-9066-4

Wagenmakers, E. J., Morey, R. D., \& Lee, M. D. (2016). Bayesian benefits for the pragmatic researcher. Current Directions in Psychological Science, 25(3), 169-176, doi: $10.1177 / 0963721416643289$

Wagenmakers, E. J., Wetzels, R., Borsboom, D., \& van der Maas, H. L. J. (2011). Why psychologists must change the way they analyze their data: The case of psi. Journal of Personality and Social Psychology, 100, 426-432, doi: 10.1037/a0022790 
Table 1. Breakdown of the verifiable source components

\begin{tabular}{lll}
\hline \multicolumn{2}{c}{ Verifiable Source } & \multicolumn{1}{c}{ Description } \\
(I) & CCTV & CCTV evidence capturing the interviewee's actions \\
(II) & Physical evidence & Physical evidence produced by the interviewee's actions (e.g. a receipt, a ticket) \\
(III) & Electronic evidence & $\begin{array}{l}\text { Electronic evidence produced by the interviewees actions, (e.g. cash } \\
\text { withdrawal, paying using a card, sent emails) }\end{array}$ \\
(IV) & Phone evidence & Sent/ received phone calls or text messages by/to the interviewee \\
(V) & Meeting other & $\begin{array}{l}\text { Deliberate meeting with another (e.g. a named friend), in principle, traceable, } \\
\text { person who can verify information }\end{array}$ \\
(VI) & Witness/ alibi & $\begin{array}{l}\text { An incidental witness who can be, in principle, traced (e.g. a named individual/ } \\
\text { an individual working at a named establishment) that can verify the information }\end{array}$
\end{tabular}


Table 2. Suggested interpretation scheme for the Bayes Factor, as proposed by Jeffrey's (1961) and modified by Wagenmakers et al. (2011)

\begin{tabular}{ll}
\hline Bayes Factor, $B F_{10}$ & Interpretation \\
\hline$>100$ & Extreme evidence for $H_{1}$ \\
$30-100$ & Very strong evidence for $H_{1}$ \\
$10-30$ & Strong evidence for $H_{1}$ \\
$3-10$ & Substantial evidence for $H_{1}$ \\
$1-3$ & Anecdotal evidence for $H_{1}$ \\
1 & No evidence \\
$1 / 3-1$ & Anecdotal evidence for $H_{0}$ \\
$1 / 10-1 / 3$ & Substantial evidence for $H_{0}$ \\
$1 / 30-1 / 10$ & Strong evidence for $H_{0}$ \\
$1 / 100-1 / 30$ & Very strong evidence for $H_{0}$ \\
$<1 / 100$ & Extreme evidence for $H_{0}$ \\
\hline
\end{tabular}


Table 3 (Study 1) Descriptive statistics for the number and proportion of verifiable detail reported in the final statement, as a function of Veracity and Preliminary questioning condition.

\begin{tabular}{lllllllll}
\hline & \multicolumn{3}{c}{ Preliminary closed questioning } & & \multicolumn{3}{c}{ Preliminary open questioning } \\
& \multicolumn{2}{c}{ Truth tellers } & \multicolumn{2}{c}{ Liars } & \multicolumn{2}{c}{ Truth tellers } \\
& Mean (SD) & $95 \%$ CI & Mean (SD) & $95 \%$ CI & Mean (SD) & $95 \%$ CI & Mean (SD) & $95 \%$ CI \\
\hline Verifiable detail (VD) & $14.00(7.27)$ & {$[10.67,17.61]$} & $7.89(7.48)$ & {$[4.53,11.70]$} & $15.76(13.75)$ & {$[10.58,23.42]$} & $2.24(2.80)$ & {$[1.47,3.83]$} \\
VD Proportion & $.32(.16)$ & {$[.24, .40]$} & $.21(.21)$ & {$[.13, .29]$} & $.33(.17)$ & {$[.25, .41]$} & $.07(.09)$ & {$[-.01, .15]$} \\
\hline
\end{tabular}


Table 4. (Study 1) Descriptive statistics for verifiable source commissions, omissions and repetitions, as a function of Veracity and Preliminary questioning condition

\begin{tabular}{|c|c|c|c|c|c|c|c|c|}
\hline & \multicolumn{4}{|c|}{ Preliminary closed questioning } & \multicolumn{4}{|c|}{ Preliminary open questioning } \\
\hline & \multicolumn{2}{|c|}{ Truth tellers } & \multicolumn{2}{|c|}{ Liars } & \multicolumn{2}{|c|}{ Truth tellers } & \multicolumn{2}{|c|}{ Liars } \\
\hline & Mean (SD) & $95 \% \mathrm{CI}$ & Mean (SD) & $95 \% \mathrm{CI}$ & Mean (SD) & $95 \% \mathrm{CI}$ & Mean (SD) & $95 \% \mathrm{CI}$ \\
\hline Verifiable source commission & $.13(.34)$ & {$[.00, .33]$} & $.35(.49)$ & {$[.13, .60]$} & $.59(.71)$ & {$[.23, .96]$} & $.00(.00)$ & $[.00, .00])$ \\
\hline Verifiable source omission & $1.75(1.18)$ & {$[1.21,2.33]$} & $1.06(1.14)$ & {$[.55,1.62]$} & $.59(.62)$ & {$[.30, .88]$} & $.59(.71)$ & {$[.29, .94]$} \\
\hline Verifiable source repetition & $1.19(.54)$ & {$[.92,1.46]$} & $.59(.71)$ & {$[.27, .93]$} & $2.29(.92)$ & {$[1.82,2.74]$} & $.47(.62)$ & {$[.21, .77]$} \\
\hline
\end{tabular}


Table 5. (Study 1) Hit Rates the number of verifiable details, and proportion of verifiable detail reported for final statement, as a function of Preliminary questioning condition.

\begin{tabular}{|c|c|c|c|c|c|c|c|}
\hline & \multicolumn{3}{|c|}{ Hit Rate } & \multirow[t]{2}{*}{$\chi^{2}$} & \multirow[t]{2}{*}{ Wilks' $\lambda$} & \multirow[t]{2}{*}{$p$-value } & \multirow[t]{2}{*}{ Canonical correlation } \\
\hline & $\begin{array}{l}\text { Truths } \\
(\%)\end{array}$ & $\begin{array}{l}\text { Lies } \\
(\%)\end{array}$ & $\begin{array}{l}\text { Total } \\
(\%)\end{array}$ & & & & \\
\hline \multicolumn{8}{|l|}{ Preliminary closed questioning condition } \\
\hline Verifiable detail (VD) & 68.8 & 70.6 & 69.7 & 5.12 & 0.85 & 0.024 & .39 \\
\hline VD proportion & 56.3 & 64.7 & 60.6 & 2.86 & 0.91 & 0.091 & .30 \\
\hline \multicolumn{8}{|c|}{ Preliminary open questioning condition } \\
\hline Verifiable detail (VD) & 58.8 & 94.1 & 76.5 & 12.65 & 0.67 & $<0.001 * * *$ & .58 \\
\hline VD proportion & 76.5 & 88.2 & 82.4 & 21.83 & 0.50 & $<0.001 * * *$ & .71 \\
\hline
\end{tabular}

Note: Accuracy rates from significant discriminant function appear in Bold

Significance level (two-tailed) $* p<.05 . * * p<.01 . * * * p<.001$. 
Table 6. (Study 2) Descriptive statistics for the number, and proportion, of verifiable detail reported in the final statement, as a function of Veracity and Preliminary questioning condition.

\begin{tabular}{|c|c|c|c|c|}
\hline & \multicolumn{2}{|c|}{ Control (IP-absent) Condition } & \multicolumn{2}{|c|}{ IP-present Condition } \\
\hline & Statement 1 & Statement 2 & Statement 1 & Statement 2 \\
\hline & $\mathrm{M}(\mathrm{SD}), 95 \% \mathrm{CI}$ & $\mathrm{M}(\mathrm{SD}), 95 \% \mathrm{CI}$ & $\mathrm{M}(\mathrm{SD}), 95 \% \mathrm{CI}$ & $\mathrm{M}(\mathrm{SD}), 95 \% \mathrm{CI}$ \\
\hline \multicolumn{5}{|l|}{ Truth Tellers } \\
\hline Verifiable detail (VD) & $14.46(11.94),[9.79,19.43]$ & 15.71 (13.18), [10.97, 21.07] & 20.29 (15.28), $[14.53,26.76]$ & $35.42(20.22),[27.17,44.04]$ \\
\hline VD Proportion & $.22(.17),[.16, .30]$ & $.25,(.18), 95 \%$ CI $[.18, .33])$ & $.28,(.15), 95 \%$ CI $[.22, .35])$ & $.42,(.18), 95 \%$ CI $[.35, .50])$ \\
\hline \multicolumn{5}{|l|}{ Liars } \\
\hline Verifiable detail (VD) & 7.08 (11.54), [3.15, 11.63] & 10.33 (18.21), [4.34, 18.87] & 8.79 (9.55), [5.30, 12.83] & 9.63 (8.99), [5.89, 13.62] \\
\hline VD Proportion & $.17(.20), 95 \%$ CI $[.10, .26])$ & $.22,(.25), 95 \%$ CI $[.13, .32])$ & $.21,(.21), 95 \%$ CI $[.13, .30])$ & $.26,(.25), 95 \%$ CI $[.16, .37])$ \\
\hline
\end{tabular}


Table 7. (Study 2) Descriptive statistics for verifiable source commissions, omissions and repetitions, as a function of Veracity and IP Condition

\begin{tabular}{|c|c|c|c|c|c|c|c|c|}
\hline & \multicolumn{4}{|c|}{ Control (IP-absent) Condition } & \multicolumn{4}{|c|}{ IP-present Condition } \\
\hline & \multicolumn{2}{|c|}{ Truth tellers } & \multicolumn{2}{|c|}{ Liars } & \multicolumn{2}{|c|}{ Truth tellers } & \multicolumn{2}{|c|}{ Liars } \\
\hline & Mean (SD) & $95 \% \mathrm{CI}$ & Mean (SD) & $95 \% \mathrm{CI}$ & Mean (SD) & $95 \% \mathrm{CI}$ & Mean (SD) & $95 \% \mathrm{CI}$ \\
\hline Verifiable source commission & $.29(.55)$ & {$[.09, .53]$} & $.38(.58)$ & {$[.16, .62]$} & $.83(.70)$ & {$[.56,1.14]$} & $.46(.93)$ & {$[.14, .88]$} \\
\hline Verifiable source omission & $.08(.28)$ & {$[.00, .22]$} & $.08(.28)$ & {$[.00, .21]$} & $.08(.28)$ & {$[.00, .23]$} & $.21(.41)$ & {$[.05, .39]$} \\
\hline Verifiable source repetition & $1.38(.71)$ & {$[1.07,1.64]$} & $.79(.78)$ & {$[.50,1.11]$} & $1.79(.83)$ & {$[1.44,2.14]$} & $.88(.90)$ & {$[.52,1.22]$} \\
\hline
\end{tabular}


Table 8. (Study 2) Hit Rates for the number of verifiable details and proportion of verifiable detail, as a function of Statement and IP Condition.

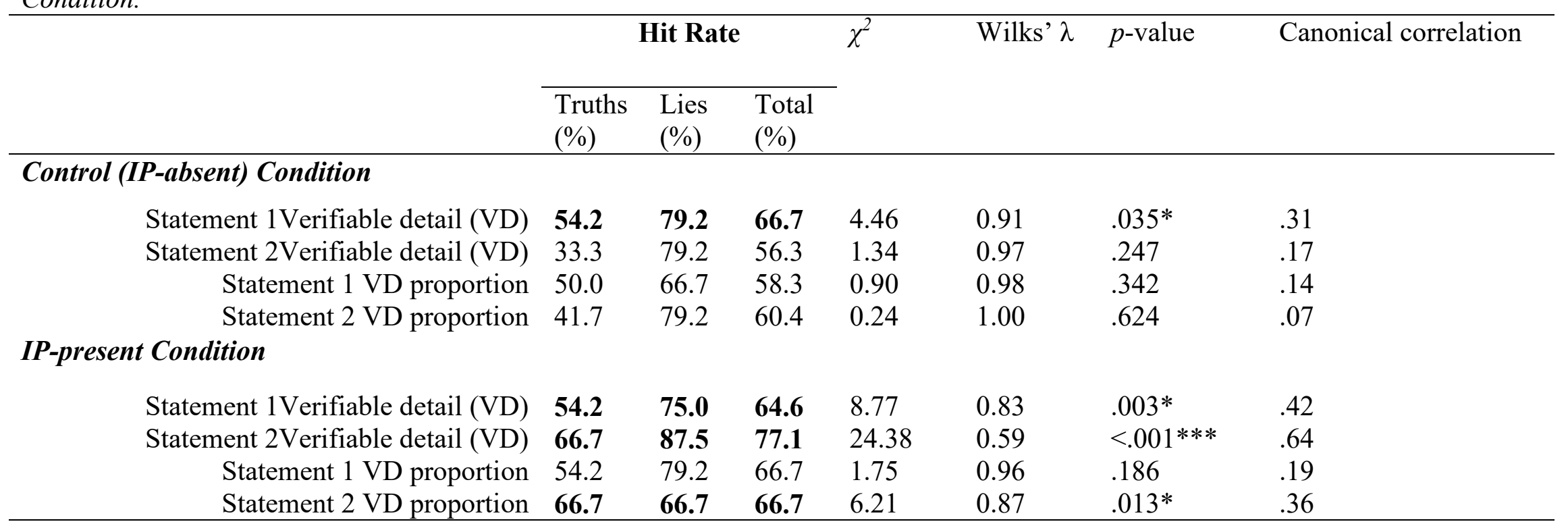

Note: Accuracy rates from significant discriminant function appear in Bold

Significance level (two-tailed) $* p<.05 . * * p<.01 . * * * p<.001$. 
Figure captions

Figure 1a: ROC curve (with AUC) for verifiable detail reported in the final statement for the preliminary closed question condition (Study 1)

Figure 1b: ROC curve (with AUC) for verifiable detail reported in the final statement for the preliminary open question condition (Study 1)

Figure 2a: ROC curve (with AUC) for the proportion of verifiable reported in the final statement for the preliminary closed question condition (Study 1)

Figure 2b: ROC curve (with AUC) for the proportion of verifiable detail in the final statement for the preliminary open question condition (Study 1) 


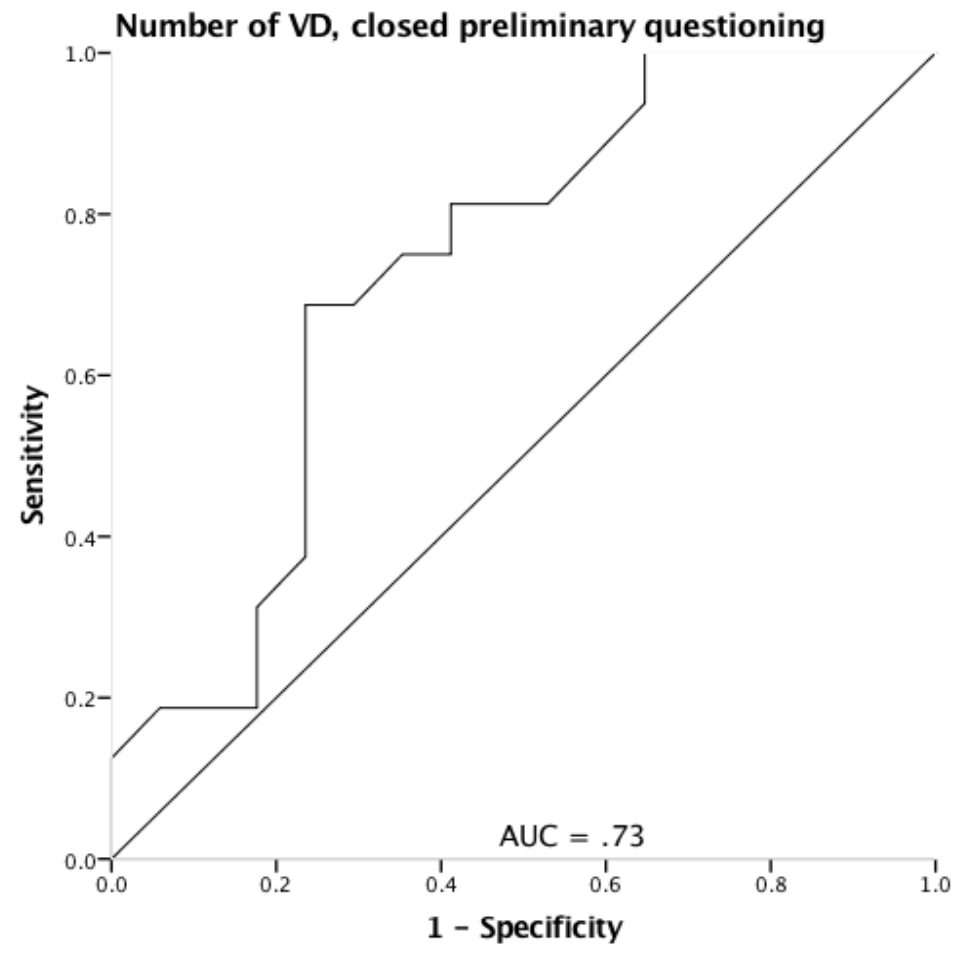

Figure 1a

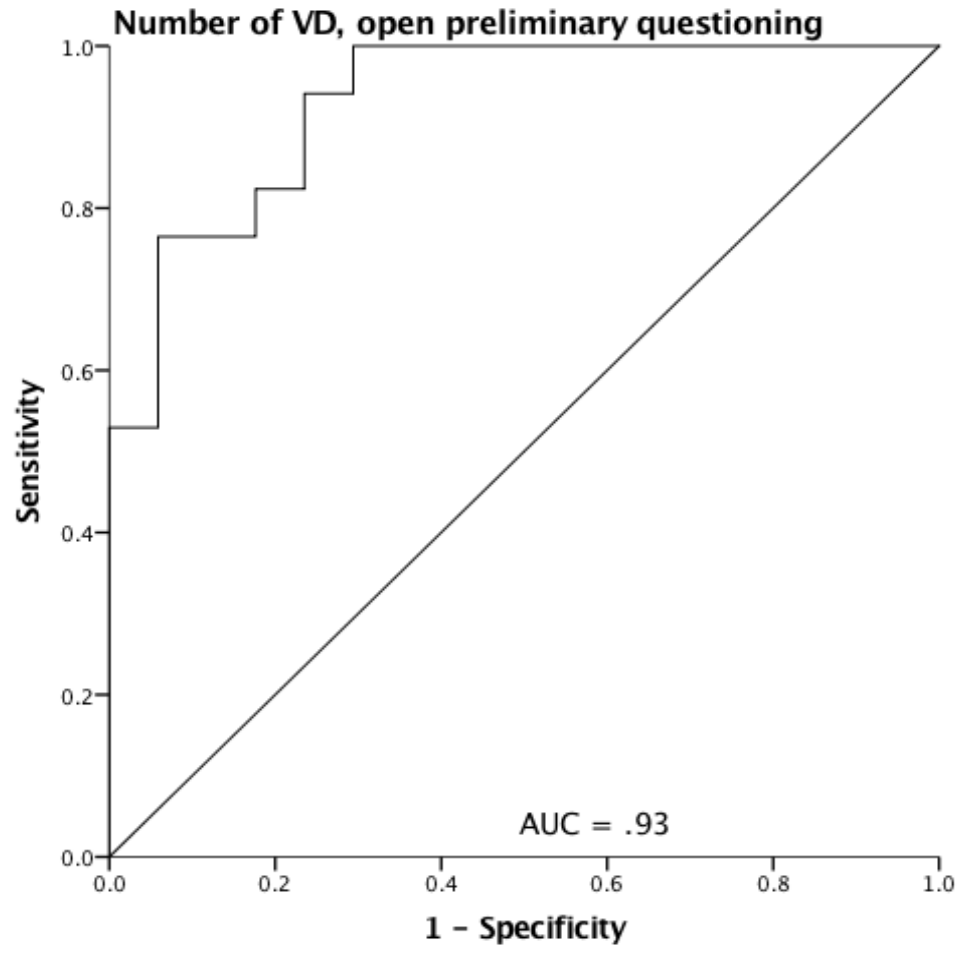

Figure 1b 


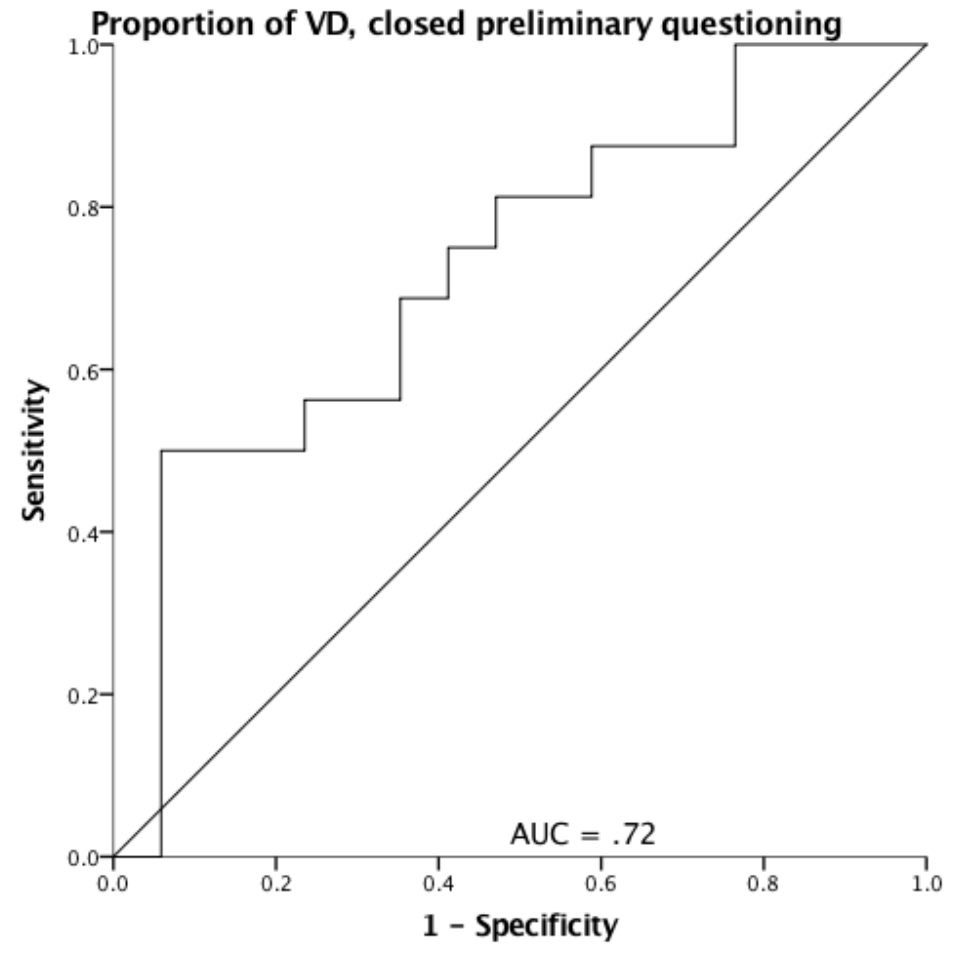

Figure 2a

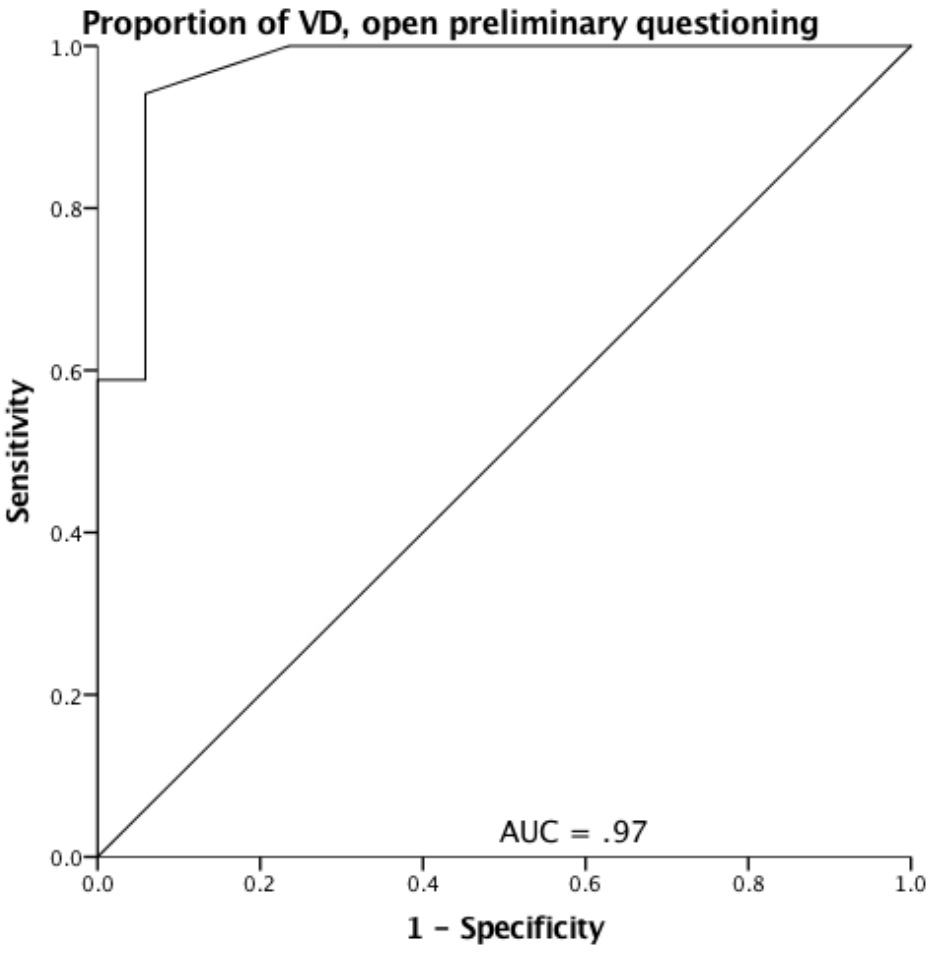

Figure $2 b$ 
Figure captions

Figure 3a: ROC curve (with AUC) for verifiable detail reported in the final statement for the Control (IP-absent) condition (Study 2)

Figure 3b: ROC curve (with AUC) for verifiable detail reported in the final statement for the IP-present condition (Study 2)

Figure 4a: ROC curve (with AUC) for the proportion of verifiable reported in the final statement for the Control (IP-absent) condition (Study 2)

Figure 4b: ROC curve (with AUC) for the proportion of verifiable detail in the final statement for the IP-present condition (Study 2) 


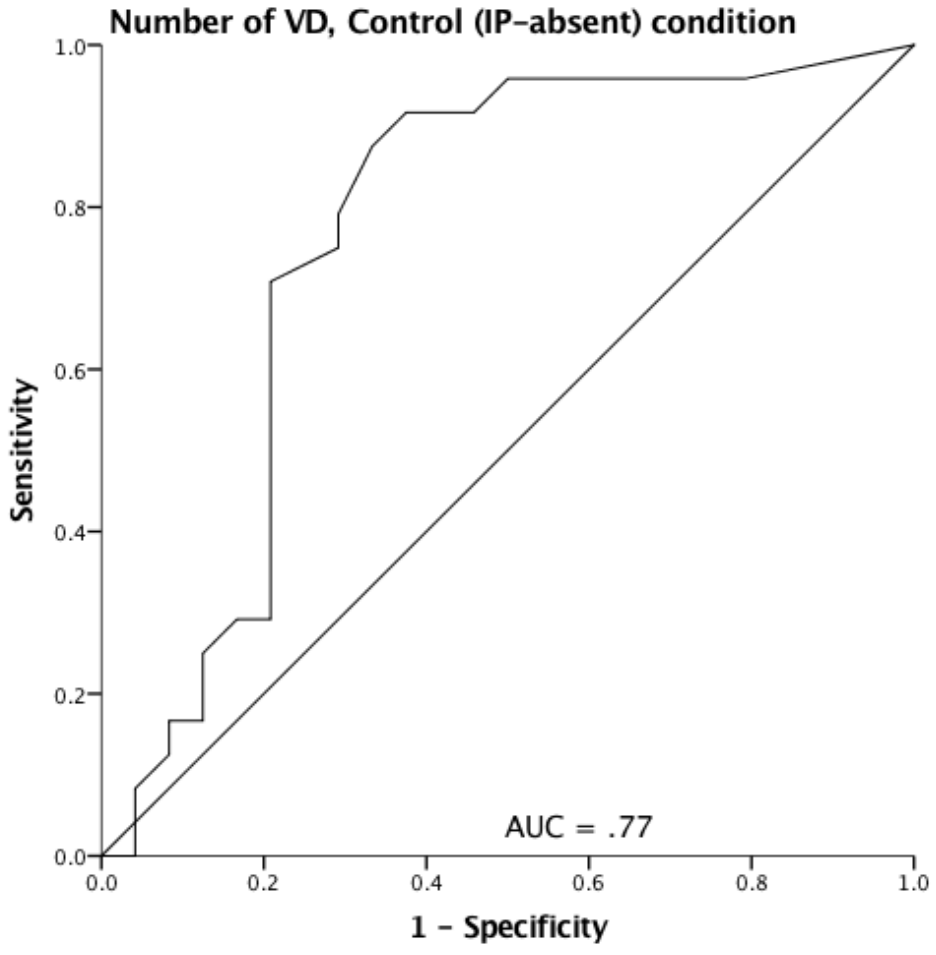

Figure 3a

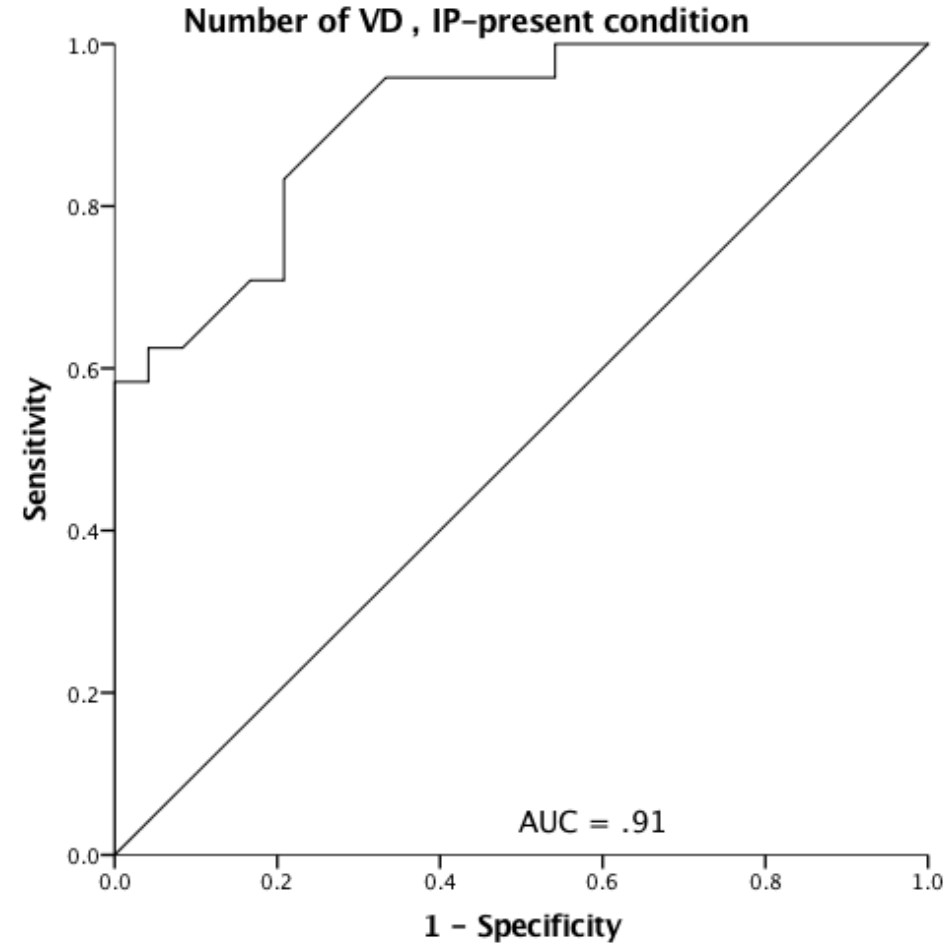

Figure $3 b$ 


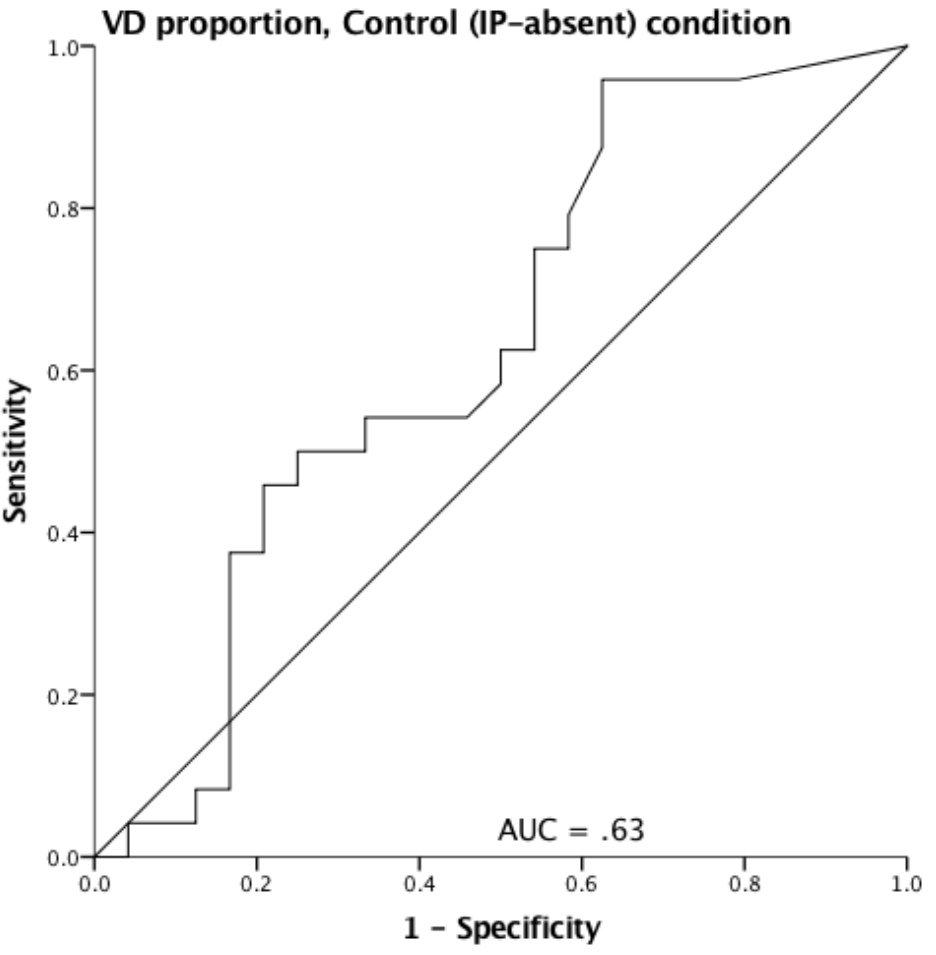

Figure $4 \mathrm{a}$

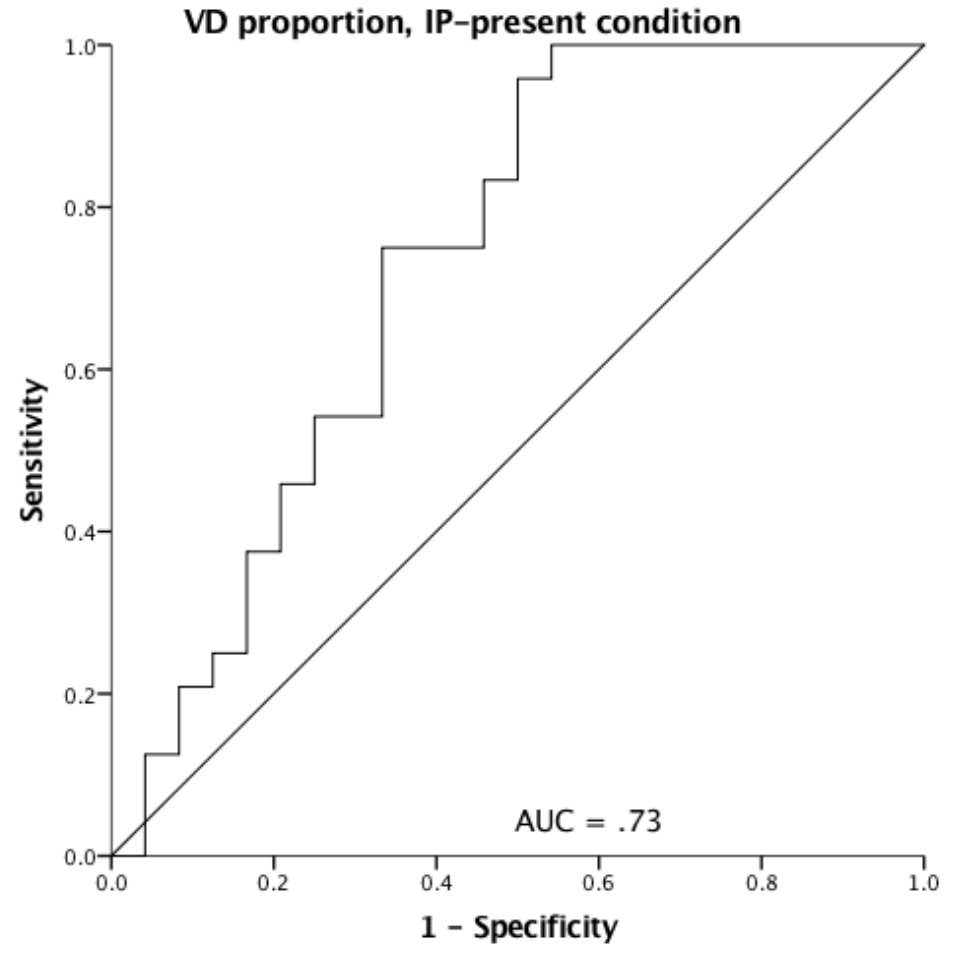

Figure $4 b$ 


\section{Appendix 1}

\section{Probe condition preliminary questioning protocol (Study 1)}

Question 1: During the 30 minutes today, did you visit a location you noticed had CCTV cameras?

Question 2: During the 30 minutes today, did you do any activity that resulted in documentation (i.e. a receipt, a ticket) that could be checked now?

Question 3: During the 30 minutes today, did you do anything that resulted in an electronic record (i.e. email someone, tweet, use a university/ bank card/ cash withdrawal from an ATM) that could be checked now?

Question 4: In the last 30 minutes today, have you received or sent a text message or phone call that could be checked now?

Question 5: In the last 30 minutes today, have you meet another person who can be identified (i.e. that you can name) and traced now?'

Question 6: In the last 30 minutes today has anyone identifiable (i.e. that you can name) witnessed your actions? 


\section{Appendix 2- Information Protocol (IP) instruction (Study 1 and 2)}

We know from research that liars prefer to avoid providing details that can be verified whereas truth tellers prefer to provide details that can be verified.

Therefore the analyst will check carefully to what extent the details you provide can be verified.

Verifiable details are i) activities carried out with identifiable or named persons who the interviewer can consult, ii) activities that have been witnessed by identifiable or named persons who the interviewer can consult, iii) activities that the interviewee believes may have been captured on CCTV and iv) activities that may have been recorded and documented, such as using debit cards, mobile phones, or computers.

The analyst may check the occurrence of some or all of the details provided in your statements after the interview to ensure their authenticity. 


\section{Supplementary analysis}

\section{Detail analysis (Study 1)}

\section{Number of unverifiable details in the final statement.}

A 2 (Veracity) x 2 (Preliminary Questioning Style) ANOVA was conducted with the number of unverifiable details in the final statement as the dependent variable. The analysis revealed no significant main effects, and the Veracity x Preliminary Questioning Style interaction effect was not significant, all $F$ 's $<2.12$, all $p$ 's $>.150$.

\section{Detail analysis (Study 2)}

\section{Number of unverifiable details reported}

A 2 (Veracity: lie/ truth) x 2 (Questioning Condition: Information Protocol/ Control) x 2 (Statement: initial/ final) mixed factors ANOVA with Veracity and Questioning Condition as the between-subject factors and Statement as the within-subjects factor was conducted using the number of reported unverifiable details as the dependent variable. This analysis revealed a main effect for Veracity, $F(1,92)=20.92, \mathrm{MSE}=28470.02, p<.001, d=.90,95 \%$ CI $[.47,1.30]$. Truth tellers $(\mathrm{M}=53.87, \mathrm{SD}=34.44,95 \% \mathrm{CI}[46.39,61.34])$ reported more unverifiable detail than liars ( $\mathrm{M}=29.51, \mathrm{SD}=16.89,95 \% \mathrm{CI}[22.03,36.99])$. No other significant main effects or interaction effects emerged, all $F$ 's $<0.62$, all $p$ 's $>.432$. 


\section{Consistency analysis of verifiable sources (Study 2)}

\section{Verifiable Source commissions}

A 2 (Veracity) x 2 (IP Condition) ANOVA was conducted with the number of reported verifiable evidence factor commissions as the dependent variable, and the number of reported verifiable evidence factors in the initial statement as the covariant. This analysis revealed a significant main effect for IP Condition, $F(1,91)=6.27, \mathrm{MSE}=3.06, p=.014, d=1.13$, $95 \%$ CI $[.68,1.54]$. However, the main effect for Veracity was not significant, $F(1,91)=$ 2.70, MSE $=1.32, p=0.104, d=.36,95 \%$ CI $[-.04, .76]$. The Veracity x IP Condition interaction effect was not significant, $F(1,91)=2.88, \mathrm{MSE}=1.41, p=0.093, \eta_{\mathrm{p}}{ }^{2}=.03$.

The interaction statistics $(p=.093)$ refers to any type of interaction. However, in Hypothesis 6 we predicted a specific type of interaction: liars will report more verifiable detail in their final statement in the preliminary closed than in the preliminary open questioning condition, whereas verifiable detail will not vary as a function of preliminary question style for truth tellers. Therefore, a more informative test of Hypothesis 6 is to statistically test for significant differences between the two Preliminary Questioning Style conditions for truth-tellers and liars separately.

No significant difference emerged for reported Verifiable Source commissions between Liars in the Control condition $(\mathrm{M}=.38, \mathrm{SD}=.58,95 \% \mathrm{CI}[.16, .62])$ and Liars in the IP Condition $(\mathrm{M}=.46, \mathrm{SD}=.93,95 \% \mathrm{CI}[.14, .88]), t(46)=.37, p=.711 d=.10,95 \% \mathrm{CI}[-.46$, $.67]$. Bayesian analysis showed our data were more in support of the null hypothesis $\left(B F_{10}=\right.$ 0.30). In contrast, truth tellers in the IP Condition $(\mathrm{M}=.83, \mathrm{SD}=.70,95 \% \mathrm{CI}[.56,1.14])$ reported significant more Verifiable Source commissions than Truth tellers in the Control Condition, $(\mathrm{M}=.29, \mathrm{SD}=.55,95 \% \mathrm{CI}[.09, .53]), t(46)=2.98, p=.005, d=.86,95 \% \mathrm{CI}[.25$, 1.43]. Bayesian analysis showed our data were more in support of the alternative hypothesis $\left(B F_{10}=8.90\right)$. These results support Hypothesis 6 . 


\section{Verifiable Source omissions.}

A 2 (Veracity) x 2 (IP Condition) ANOVA was conducted with the number of reported verifiable evidence factor omissions as the dependent variable, and the number of reported verifiable evidence factors in the initial statement as the covariant. This analysis revealed no significant main effect for IP Condition, $F(1,91)=.33$, MSE $=.03, p=.568, d=.12,95 \%$ CI $[-.28, .52]$, and no significant main effect for Veracity, $F(1,91)=2.60$, MSE $=2.61, p=$ $0.111, d=.36,95 \%$ CI $[-.05, .75]$. Furthermore, the Veracity x IP Condition interaction effect was not significant, $F(1,91)=1.12, \mathrm{MSE}=.11, p=0.292, \eta_{\mathrm{p}}{ }^{2}=.01$

\section{Verifiable Source repetitions.}

A 2 (Veracity) x 2 (IP Condition) ANOVA was conducted with the number of reported verifiable evidence factor omissions as the dependent variable, and the number of reported verifiable evidence factors in the initial statement as the covariant. This analysis revealed no significant main effect for IP Condition, $F(1,91)=.72$, MSE $=.08, p=.397, d=.14,95 \%$ CI $[-.27, .53]$, and no significant main effect for Veracity, $F(1,91)=1.478$, MSE $=.17, p=$ $0.227, d=.11,95 \%$ CI $[-.29, .51]$. Furthermore, the Veracity x IP Condition interaction effect was not significant, $F(1,91)=1.68, \mathrm{MSE}=.19, p=0.198, \eta_{\mathrm{p}}{ }^{2}=.02$.

\section{Consistency analysis of unverifiable detail (Study 2)}

\section{Unverifiable commissions}

A 2 (Veracity: lie/ truth) x 2 (Questioning Condition: Information Protocol/ Control) between-subject ANOVA was conducted using the number of unverifiable commissions as the dependent variable, and with the number of unverifiable details reported in statement 1 as the covariant. No significant main effects emerged and the Veracity x Questioning condition interaction was not significant, all $F$ 's $<.53$, all $p$ 's $>.471$.

\section{Unverifiable omissions}


A 2 (Veracity: lie/ truth) x 2 (Questioning Condition: Information Protocol/ Control) between-subject ANOVA was conducted using the number of unverifiable omissions as the dependent variable, and with the number of unverifiable details reported in statement 1 as the covariant. No significant main effects emerged and the Veracity x Questioning condition interaction was not significant, all $F$ 's $<1.29$, all $p$ 's $>.259$.

\section{Unverifiable repetitions}

A 2 (Veracity: lie/ truth) x 2 (Questioning Condition: Information Protocol/ Control) between-subject ANOVA was conducted using the number of unverifiable repetitions as the dependent variable, and with the number of unverifiable details reported in statement 1 as the covariant. No significant main effects emerged and the Veracity x Questioning condition interaction was not significant, all $F^{\prime}$ s $<1.29$, all $p$ 's $>.259$. 